\title{
Coupling of symmetric operators and the third Green identity
}

\author{
Jussi Behrndt $^{1}$ - Vladimir Derkach ${ }^{2}$. \\ Fritz Gesztesy ${ }^{3,4}$. Marius Mitrea ${ }^{3}$
}

Received: 13 March 2017 / Revised: 21 March 2017 / Accepted: 21 March 2017 /

Published online: 30 March 2017

(C) The Author(s) 2017. This article is an open access publication

\begin{abstract}
The principal aim of this paper is to derive an abstract form of the third Green identity associated with a proper extension $T$ of a symmetric operator $S$ in a Hilbert space $\mathfrak{H}$, employing the technique of quasi boundary triples for $T$. The general
\end{abstract}

\section{Communicated by Ari Laptev.}

J. B. gratefully acknowledges financial support by the Austrian Science Fund (FWF), Project P 25162-N26; M. M. is indebted to the Simons Foundation for support under Grant No. 281566; V. D. is indebted to the Fulbright Fund for support under Grant No. 68130028.

$凶$ Fritz Gesztesy

Fritz_Gesztesy@baylor.edu

http://www.baylor.edu/math/index.php?id=935340

Jussi Behrndt

behrndt@tugraz.at

http://www.math.tugraz.at/ behrndt/

Vladimir Derkach

derkach.v@gmail.com

Marius Mitrea

mitream@missouri.edu

http://www.math.missouri.edu/personnel/faculty/mitream.html

1 Institut für Numerische Mathematik, Technische Universität Graz, Steyrergasse 30, 8010 Graz, Austria

2 Department of Mathematics, Dragomanov National Pedagogical University, Pirogova 9, Kiev 01601, Ukraine

3 Department of Mathematics, University of Missouri, Columbia, MO 65211, USA

4 Present Address: Department of Mathematics, Baylor University, One Bear Place \#97328, Waco, TX 76798-7328, USA 
results are illustrated with couplings of Schrödinger operators on Lipschitz domains on smooth, boundaryless, compact Riemannian manifolds.

Keywords Symmetric operators - Self-adjoint extensions - Generalized resolvents · Boundary triples · Weyl-Titchmarsh functions · Schrödinger operator ·

Lipschitz domain

Mathematics Subject Classification Primary 47A10 - 47A57 - 47B25 - 58J05 . 58J32; Secondary 47A55 $\cdot$ 47B15 $\cdot$ 58J50

\section{Contents}

1 Introduction

2 Quasi boundary triples and their Weyl functions

3 The coupling model

4 The third Green identity

5 Coupling of Schrödinger operators on Lipschitz domains on manifolds

References

\section{Introduction}

The work in this paper is motivated in part by the following innocuous question: How to rule out that a Dirichlet eigenfunction of the Laplacian, or, more generally, a uniformly elliptic second order partial differential operator on a bounded domain $\Omega \subset \mathbb{R}^{n}, n \in \mathbb{N}$, is also simultaneously a Neumann eigenfunction?

Several lines of attack are available, depending on the regularity assumptions made on the underlying domain. For instance, in the case of the Dirichlet Laplacian on a bounded Lipschitz domain $\Omega \subset \mathbb{R}^{n}$, if

$$
-\Delta u=\lambda u, \quad u \uparrow \Omega=0, \quad u \in H^{1}(\Omega), \quad u \neq \equiv 0,
$$

then $u$ belongs to $H^{1}(\Omega)$, the normal derivative $\partial u / \partial v$ is square-integrable on $\partial \Omega$ (see [4]), and Rellich's identity (established in [36] for smooth domains, which nonetheless continues to be valid in the present setting) for the Dirichlet eigenvalue $\lambda$ reads

$$
\lambda=\frac{1}{4\|u\|_{L^{2}(\Omega)}^{2}} \int_{\partial \Omega}\left(\frac{\partial u}{\partial v}(\xi)\right)^{2}\left(\frac{\partial|x|^{2}}{\partial v}(\xi)\right) d^{n-1} \omega(\xi) .
$$

Here $x=\left(x_{1}, \ldots, x_{n}\right) \in \mathbb{R}^{n}, d^{n-1} \omega$ denotes the surface measure on $\partial \Omega, v$ is the outward pointing unit normal vector at points of $\partial \Omega$, and $\partial / \partial v$ represents the normal derivative,

$$
\frac{\partial}{\partial v}:=v(\xi) \cdot \nabla_{\xi}, \quad \xi \in \partial \Omega
$$

Thus, vanishing of the normal derivative (i.e., the Neumann boundary condition) $\partial u / \partial v=0$ on $\partial \Omega$ yields $\lambda=0$ which contradicts the well-known fact that the 
Dirichlet Laplacian is strictly positive on bounded (in fact, finite Euclidean volume) domains $\Omega$.

A second approach is based on the fact that if $\Omega \subset \mathbb{R}^{n}$ is a bounded Lipschitz domain then (see [32, Corollary 3.21, p. 161] for more general results of this flavor)

$$
\stackrel{\circ}{H}^{2}(\Omega)=\left\{v \in H^{2}(\Omega) \mid u\left\lceil\partial \Omega=\left(\partial_{\nu} u\right)\lceil\partial \Omega=0\} .\right.\right.
$$

In turn, this may be used to show that, whenever $\Omega \subset \mathbb{R}^{n}$ is a bounded Lipschitz domain, if

$$
\left.-\Delta u=\lambda u, \quad u \curlyvee \Omega=\left(\partial_{\nu} u\right)\right\rceil \Omega=0, u \in H^{1}(\Omega),
$$

then $u$ necessarily vanishes. Specifically, (1.5) first implies (see [4]) that $u \in H^{2}(\Omega)$ which, together with (1.4), proves that the extension $\tilde{u}$ of $u$ to $\mathbb{R}^{n}$ by zero outside $\Omega$ lies in $H^{2}\left(\mathbb{R}^{n}\right)$ and satisfies $-\Delta \widetilde{u}=\lambda \tilde{u}$ in $\mathbb{R}^{n}$. As such, if $u$ were not identically zero in $\Omega$, the Laplacian in $\mathbb{R}^{n}$ would have a compactly supported eigenfunction, clearly a contradiction. This rules out the eventuality of a Dirichlet eigenfunction simultaneously being a Neumann eigenfunction. An argument of this nature may be adapted to more general uniformly elliptic second order partial differential operators via unique continuation principles; see, for instance, [2,3,20,23, 25, 26,38].

An approach, intimately related to the one just discussed, adding a functional analytic flavor, would employ the fact that if $\Omega \subset \mathbb{R}^{n}$ is a bounded Lipschitz domain then the Dirichlet and Neumann Laplacians in $L^{2}(\Omega)$, denoted by $-\Delta_{D, \Omega}$ and $-\Delta_{N, \Omega}$, respectively, are relatively prime and hence satisfy

$$
\operatorname{dom}\left(-\Delta_{D, \Omega}\right) \cap \operatorname{dom}\left(-\Delta_{N, \Omega}\right)=\stackrel{\circ}{H}^{2}(\Omega)=\operatorname{dom}\left(-\Delta_{\min , \Omega}\right) .
$$

Here the associated minimal and maximal operators in $L^{2}(\Omega)$ are of the form

$$
\begin{aligned}
& -\Delta_{\min , \Omega}=-\Delta, \quad \operatorname{dom}\left(-\Delta_{\min , \Omega}\right)=\stackrel{\circ}{H}^{2}(\Omega), \\
& -\Delta_{\max , \Omega}:=-\Delta, \quad \operatorname{dom}\left(-\Delta_{\max , \Omega}\right):=\left\{f \in L^{2}(\Omega) \mid \Delta f \in L^{2}(\Omega)\right\},
\end{aligned}
$$

where the expression $\Delta f, f \in L^{2}(\Omega)$, is understood in the sense of distributions. In addition, one has the relations

$$
-\Delta_{\min , \Omega}^{*}=-\Delta_{\max , \Omega}, \quad-\Delta_{\min , \Omega}=-\Delta_{\max , \Omega}^{*}
$$

(see, for instance, [4, Sect. 3]). Invoking the fact that the minimal operator $-\Delta_{\min , \Omega}$ is simple (i.e., it has no invariant subspace on which it is self-adjoint), and simple operators have no eigenvalues, $-\Delta_{\min , \Omega}$ cannot have any eigenvalues, thus, no nonzero solution $u$ satisfying (1.5) exists. For recent results of this type see, for instance, [9, Proposition 2.5]. Upon modifications employing appropriate Dirichlet and Neumann traces this approach remains applicable to the more general case of uniformly elliptic second order partial differential operators on Lipschitz domains $\Omega$ (see, e.g., [4,9]).

Perhaps, a most illuminating proof of the impossibility of a Dirichlet eigenfunction to be simultaneously a Neumann eigenfunction can be based on the third Green identity, which naturally leads to one of the principal topics of this paper. Assuming again $\Omega$ 
to be a bounded Lipschitz domain in $\mathbb{R}^{n}$, we note the following well-known special case of the third Green identity (see, e.g., [12], [24, p. 97], [29, Theorem 6.10]),

$$
\begin{aligned}
& u(x)=\left(\mathcal{G}_{z}(-\Delta-z) u\right)(x)-\left(\mathcal{D}_{z}\left(u \Upsilon_{\partial \Omega}\right)\right)(x)+\left(\mathcal{S}_{z}\left(\partial_{\nu} u \uparrow_{\partial \Omega}\right)\right)(x), \\
& u \in H^{2}(\Omega), \quad z \in \mathbb{C}, \quad x \in \Omega
\end{aligned}
$$

in terms of the resolvent operator $\mathcal{G}_{z}$ for $-\Delta$ defined on $H^{2}\left(\mathbb{R}^{n}\right)$, and the single and double layer potentials $\mathcal{S}_{z}$ and $\mathcal{D}_{z}, z \in \mathbb{C}$, defined by

$$
\begin{aligned}
& \left(\mathcal{G}_{z} f\right)(x)=\int_{\mathbb{R}^{n}} E_{n}^{(0)}(z ; x-y) f(y) d^{n} y, \quad f \in L^{2}\left(\mathbb{R}^{n}\right), x \in \mathbb{R}^{n} \\
& \left(\mathcal{S}_{z} v\right)(x)=\int_{\partial \Omega} E_{n}^{(0)}(z ; x-\xi) v(\xi) d^{n-1} \omega(\xi), \quad v \in L^{2}(\partial \Omega), \quad x \in \Omega, \\
& \left(\mathcal{D}_{z} v\right)(x)=\int_{\partial \Omega}\left(\partial_{\nu(\xi)} E_{n}^{(0)}\right)(z ; x-\xi) v(\xi) d^{n-1} \omega(\xi), \quad v \in L^{2}(\partial \Omega), \quad x \in \Omega .1
\end{aligned}
$$

Here $E_{n}^{(0)}(z ; x)$ represents the standard fundamental solution of the Helmholtz differential expression $-\Delta-z$ in $\mathbb{R}^{n}, n \in \mathbb{N}, n \geq 2$, that is,

$$
E_{n}^{(0)}(z ; x)= \begin{cases}(i / 4)\left(2 \pi|x| / z^{1 / 2}\right)^{(2-n) / 2} H_{(n-2) / 2}^{(1)}\left(z^{1 / 2}|x|\right), & n \geq 2, \quad z \in \mathbb{C} \backslash\{0\}, \\ \frac{-1}{2 \pi} \ln (|x|), & n=2, \quad z=0 \\ \frac{1}{(n-2) \omega_{n-1}}|x|^{2-n}, & n \geq 3, \quad z=0 \\ \operatorname{Im}\left(z^{1 / 2}\right) \geq 0, & x \in \mathbb{R}^{n} \backslash\{0\}, \quad(1.14)\end{cases}
$$

where $H_{v}^{(1)}(\cdot)$ denotes the Hankel function of the first kind with index $v \geq 0$ (cf. [1, Sect. 9.1]).

Thus, if $u$ is assumed to satisfy (1.5) then, as already noted, $u \in H^{2}(\Omega)$ so the third Green identity (1.10) instantly yields $u=0$ in $\Omega$, hence the nonexistence of nontrivial solutions $u$ satisfying (1.5). Again, this approach extends to the more general case of uniformly elliptic second order partial differential operators $\mathcal{L}$ by appropriately replacing in (1.10)-(1.14) the fundamental solution $E_{n}^{(0)}(z ; x-y)$ for the Helmholtz operator $-\Delta-z$ in $\mathbb{R}^{n}$ by the fundamental solution $E_{n}(z ; x-y)$ of $\mathcal{L}-z$ in $\mathbb{R}^{n}$.

The approach based on Green's third identity just outlined has several features. First, while the original issue pertaining to properties of eigenfunctions of the Dirichlet Laplacian $-\Delta_{D, \Omega}$ in $L^{2}(\Omega)$ may be formulated entirely in terms on $\Omega$ and $\partial \Omega$, the third Green identity (1.10) naturally involves the fundamental solution $E_{n}^{(0)}(z ; x-y)$ for the Laplacian in $\mathbb{R}^{n}$. The latter is obviously independent of $\Omega$ and $\partial \Omega$.

Second, denoting

$$
\Omega_{+}:=\Omega, \quad \Omega_{-}:=\mathbb{R}^{n} \backslash \bar{\Omega},
$$

one is naturally led to a comparison of the Dirichlet Laplacian

$$
\left(-\Delta_{D, \Omega_{+}}\right) \oplus\left(-\Delta_{D, \Omega_{-}}\right) \text {in } L^{2}\left(\Omega_{+}\right) \oplus L^{2}\left(\Omega_{-}\right) \simeq L^{2}\left(\mathbb{R}^{n}\right)
$$


and the Laplacian $-\Delta$ in $L^{2}\left(\mathbb{R}^{n}\right)$ (with domain $H^{2}\left(\mathbb{R}^{n}\right)$ ). While the Dirichlet Laplacian $\left(-\Delta_{D, \Omega_{+}}\right) \oplus\left(-\Delta_{D, \Omega_{-}}\right)$corresponds to a complete decoupling of $\mathbb{R}^{n}$ into $\Omega_{+} \cup \Omega_{-}$ (ignoring the compact boundary $\mathcal{C}:=\partial \Omega_{ \pm}$of $n$-dimensional Lebesgue measure zero), in stark contrast to this decoupling, the Laplacian $-\Delta$ on $H^{2}\left(\mathbb{R}^{n}\right)$ couples $\Omega_{+}$and $\Omega_{-}$via the imposition of continuity conditions across $\mathcal{C}$ of the form

$$
u_{+}\left\lceil\partial \Omega_{+}=u_{-}\left\lceil\partial \Omega_{-}, \quad-\partial_{\nu} u_{+}\left\lceil\partial \Omega_{+}=\partial_{\nu} u_{-}\left\lceil\partial \Omega_{-} .\right.\right.\right.\right.
$$

Here we identified $u \in L^{2}\left(\mathbb{R}^{n}\right)$ with the pair $\left(u_{+}, u_{-}\right) \in L^{2}\left(\Omega_{+}\right) \oplus L^{2}\left(\Omega_{-}\right)$via $u_{ \pm}=u\left\lceil\Omega_{ \pm}\right.$. The relative sign change in the normal derivatives in the second part of (1.17) is of course being a side-effect of the opposite orientations of the outward unit normals to $\Omega_{ \pm}$at points in $\mathcal{C}=\partial \Omega_{ \pm}$.

It is this coupling of $\Omega_{+}$and $\Omega_{-}$through their joint boundary $\mathcal{C}$ via the Laplacian $-\Delta$ on $\mathbb{R}^{n}$ via the continuity requirements (1.17) which constitutes the second major topic in this paper. In fact, from this point of view, the exterior domain $\Omega_{-}$plays a similar role as the original domain $\Omega=\Omega_{+}$(apart from being unbounded). Moreover, introducing the jumps of $u$ and $\partial_{\nu} u$ across $\mathcal{C}=\partial \Omega_{ \pm}$as

$$
[u]:=-u_{+}\left\lceil\partial \Omega_{+}+u_{-}\left\lceil\partial \Omega_{-}, \quad\left[\partial_{\nu} u\right]:=-\partial_{\nu} u_{+}\left\lceil\partial \Omega_{+}-\partial_{\nu} u_{-}\left\lceil\partial \Omega_{-},\right.\right.\right.\right.
$$

the third Green identity (1.10) can be shown to acquire the following form (which is symmetric with respect to $\Omega_{ \pm}$; cf., e.g., [12], [29, Theorem 6.10]):

$$
\begin{aligned}
u(x) & =\left(\mathcal{G}_{z}(-\Delta-z) u\right)(x)+\left(\mathcal{D}_{z}[u]\right)(x)-\left(\mathcal{S}_{z}\left(\left[\partial_{v} u\right]\right)\right)(x), \\
u & =\left(u_{+}, u_{-}\right), u_{ \pm} \in H^{2}\left(\Omega_{ \pm}\right), \operatorname{supp}(u) \text { compact, } z \in \mathbb{C}, x \in \mathbb{R}^{n} \backslash \mathcal{C} .
\end{aligned}
$$

At this point we are prepared to describe the major objectives of this paper: Decompose a given complex, separable Hilbert space $\mathfrak{H}$ into an orthogonal sum of closed subspaces $\mathfrak{H}_{ \pm}$as $\mathfrak{H}=\mathfrak{H}_{+} \oplus \mathfrak{H}_{-}$, consider densely defined, closed symmetric operators $S_{ \pm}$in $\mathfrak{H}_{ \pm}$and their direct sum $S=S_{+} \oplus S_{-}$in $\mathfrak{H}$, introduce restrictions $T_{ \pm}$of $S_{ \pm}^{*}$ such that $\overline{T_{ \pm}}=S_{ \pm}^{*}$ and appropriate restrictions $A_{0, \pm}$ of $T_{ \pm}$, for instance, $A_{0, \pm}$ selfadjoint in $\mathfrak{H}_{ \pm}$, defined in terms of certain abstract boundary conditions, and then find a self-adjoint operator $A$ in $\mathfrak{H}$ which closely resembles $A_{0}=A_{0,+} \oplus A_{0,-}$, but without any remnants of the boundary conditions in $A_{0,+} \oplus A_{0,-}$ and without any reference to the decomposition of $\mathfrak{H}$ into $\mathfrak{H}_{+} \oplus \mathfrak{H}_{-}$(i.e., $A$ naturally couples $\mathfrak{H}_{ \pm}$in terms of certain continuity requirements through an abstract "boundary"). Finally, derive an abstract third Green identity invoking the resolvent (or fundamental solution operator $\mathcal{G}$ ) of $A$, the operator $T=T_{+} \oplus T_{-}$, and abstract single and double layer operators constructed from $\mathcal{G}$. This can indeed be achieved with the help of an appropriate quasi boundary triple for $T$ which also permits one to introduce a natural abstract analog of the "boundary Hilbert space" $L^{2}(\mathcal{C})$ in the concrete case of the Laplacian above.

In Sect. 2 we briefly recall the basic setup for quasi boundary triples and associated operator-valued Weyl-functions (also called Weyl-Titchmarsh functions) as needed in this paper. The introduction of quasi boundary triples is intimately connected with an 
abstract (second) Green identity. Section 3 studies the operator $A$ and derives Kreintype resolvent formulas for it in terms of $A_{0}$ and a related operator. Section 4 derives the abstract third Green identity, and finally Sect. 5 illustrates the abstract material in Sects. 2-4 with the concrete case of Schrödinger operators on Lipschitz domains on smooth, boundaryless, compact Riemannian manifolds.

Finally, we briefly summarize the basic notation used in this paper: Let $\mathcal{H}, \mathfrak{H}$ be separable complex Hilbert spaces, $(\cdot, \cdot)_{\mathcal{H}}$ the scalar product in $\mathcal{H}$ (linear in the second factor), and $I_{\mathcal{H}}$ the identity operator in $\mathcal{H}$. If $T$ is a linear operator mapping (a subspace of ) a Hilbert space into another, $\operatorname{dom}(T)$ denotes the domain of $T$. The closure of a closable operator $S$ is denoted by $\bar{S}$. The spectrum and resolvent set of a closed linear operator in $\mathcal{H}$ will be denoted by $\sigma(\cdot)$ and $\rho(\cdot)$, respectively. The Banach space of bounded linear operators in $\mathcal{H}$ is denoted by $\mathcal{B}(\mathcal{H})$; in the context of two Hilbert spaces, $\mathcal{H}_{j}, j=1,2$, we use the analogous abbreviation $\mathcal{B}\left(\mathcal{H}_{1}, \mathcal{H}_{2}\right)$. The set of all closed linear operators in $\mathcal{H}$ is denoted by $\mathcal{C}(\mathcal{H})$. Moreover, $\mathcal{X}_{1} \hookrightarrow \mathcal{X}_{2}$ denotes the continuous embedding of the Banach space $\mathcal{X}_{1}$ into the Banach space $\mathcal{X}_{2}$. We also abbreviate $\mathbb{C}_{ \pm}:=\{z \in \mathbb{C} \mid \operatorname{Im}(z) \gtrless 0\}$.

\section{Quasi boundary triples and their Weyl functions}

In this section we briefly recall the notion of quasi boundary triples and the associated (operator-valued) Weyl functions.

In the following let $S$ be a densely defined closed symmetric operator in $\mathfrak{H}$.

Definition 2.1 Let $T \subseteq S^{*}$ be a linear operator in $\mathfrak{H}$ such that $\bar{T}=S^{*}$. A triple $\left\{\mathcal{H}, \Gamma_{0}, \Gamma_{1}\right\}$ is called a quasi boundary triple for $T \subseteq S^{*}$ if $\left(\mathcal{H},(\cdot, \cdot)_{\mathcal{H}}\right)$ is a Hilbert space and $\Gamma_{0}, \Gamma_{1}: \operatorname{dom}(T) \rightarrow \mathcal{H}$ are linear mappings such that the following items (i)-(iii) hold:

(i) The abstract (second) Green identity

$$
(T f, g)_{\mathfrak{H}}-(f, T g)_{\mathfrak{H}}=\left(\Gamma_{1} f, \Gamma_{0} g\right)_{\mathcal{H}}-\left(\Gamma_{0} f, \Gamma_{1} g\right)_{\mathcal{H}}, \quad f, g \in \operatorname{dom}(T),
$$

is valid.

(ii) The map $\Gamma=\left(\Gamma_{0}, \Gamma_{1}\right)^{\top}: \operatorname{dom}(T) \rightarrow \mathcal{H}^{2}$ has dense range.

(iii) $A_{0}:=T \uparrow \operatorname{ker}\left(\Gamma_{0}\right)$ is a self-adjoint operator in $\mathfrak{H}$.

The notion of quasi boundary triples was introduced in [5] and extends the concepts of ordinary (and generalized) boundary triples, see, for instance, [11,19,21,27], and the references therein. We recall that the triple in Definition 2.1 is called an ordinary boundary triple (generalized boundary triple) if item (ii) is replaced by the condition $\operatorname{ran}(\Gamma)=\mathcal{H}^{2}\left(\operatorname{ran}\left(\Gamma_{0}\right)=\mathcal{H}\right.$, respectively). On the other hand, the notion of quasi boundary triple is a particular embodiment of the more general notion of isometric/unitary boundary triples. The latter goes back to Calkin [13] and was studied in detail in $[15,17]$.

We recall briefly some important properties of quasi boundary triples. First of all, we note that a quasi boundary triple for $S^{*}$ exists if and only if the defect numbers

$$
n_{ \pm}(S)=\operatorname{dim}\left(\operatorname{ker}\left(S^{*} \mp i\right)\right)
$$


of $S$ are equal. Second, assume that $\left\{\mathcal{H}, \Gamma_{0}, \Gamma_{1}\right\}$ is a quasi boundary triple for $T \subseteq S^{*}$. Then the mapping $\Gamma=\left(\Gamma_{0}, \Gamma_{1}\right)^{\top}: \operatorname{dom}(T) \rightarrow \mathcal{H}^{2}$ is closable and $\operatorname{ker}(\Gamma)=\operatorname{dom}(S)$ holds (cf. [5, Proposition 2.2]). Third, according to [5, Theorem 2.3] one has $T=$ $S^{*}$ if and only if $\operatorname{ran}(\Gamma)=\mathcal{H}^{2}$, in this case the restriction $A_{0}=S^{*} \uparrow \operatorname{ker}\left(\Gamma_{0}\right)$ is automatically self-adjoint and the the quasi boundary triple $\left\{\mathcal{H}, \Gamma_{0}, \Gamma_{1}\right\}$ is an ordinary boundary triple in the usual sense. In this context we also note that in the case of finite deficiency indices of $S$ a quasi boundary triple is automatically an ordinary boundary triple.

Next, the notion of the $\gamma$-field and Weyl function associated to a quasi boundary triple will be recalled. First, one observes that for each $z \in \rho\left(A_{0}\right)$, the direct sum decomposition

$$
\operatorname{dom}(T)=\operatorname{dom}\left(A_{0}\right) \dot{+} \operatorname{ker}\left(T-z I_{\mathfrak{H}}\right)=\operatorname{ker}\left(\Gamma_{0}\right) \dot{+} \operatorname{ker}\left(T-z I_{\mathfrak{H}}\right)
$$

holds. Hence the restriction of the mapping $\Gamma_{0}$ to $\operatorname{ker}\left(T-z I_{\mathfrak{H}}\right)$ is injective and its range coincides with $\operatorname{ran}\left(\Gamma_{0}\right)$.

Definition 2.2 Let $\left\{\mathcal{H}, \Gamma_{0}, \Gamma_{1}\right\}$ be a quasi boundary triple for $T \subseteq S^{*}$. The $\gamma$-field $\gamma$ and the Weyl function $M$ corresponding to $\left\{\mathcal{H}, \Gamma_{0}, \Gamma_{1}\right\}$ are defined by

$$
\rho\left(A_{0}\right) \ni z \mapsto \gamma(z):=\left(\Gamma_{0} \uparrow \operatorname{ker}\left(T-z I_{\mathfrak{H}}\right)\right)^{-1}
$$

and

$$
\rho\left(A_{0}\right) \ni z \mapsto M(z):=\Gamma_{1} \gamma(z)
$$

respectively.

The notions of $\gamma$-field and Weyl function corresponding to ordinary and generalized boundary triples have been introduced in $[18,19]$, respectively; the definition above is formally the same. In the special case of ordinary and generalized boundary triples the Weyl function $M$ turns out to be a Herglotz-Nevanlinna function with values in $\mathcal{B}(\mathcal{H})$, that is, $M$ is holomorphic on $\mathbb{C} \backslash \mathbb{R}$, and

$$
\operatorname{Im}(z) \operatorname{Im}(M(z)) \geqslant 0 \text { and } M(z)=M(\bar{z})^{*}, \quad z \in \mathbb{C} \backslash \mathbb{R} \text {. }
$$

The values of the $\gamma$-field are bounded operators from $\mathcal{H}$ into $\mathfrak{H}$ with $\operatorname{ran}(\gamma(z))=$ $\operatorname{ker}\left(T-z I_{\mathfrak{H}}\right)$ and the following identity holds

$$
M(z)-M(z)^{*}=(z-\bar{z}) \gamma(z)^{*} \gamma(z), \quad z \in \rho\left(A_{0}\right)
$$

In the case of a quasi boundary triple the operators $\gamma(z), z \in \rho\left(A_{0}\right)$, are defined on the dense subspace $\operatorname{ran}\left(\Gamma_{0}\right) \subseteq \mathcal{H}$ and map onto $\operatorname{ker}\left(T-z I_{\mathfrak{H}}\right) \subset \mathfrak{H}$. By $[5$, Proposition 2.6] the operator $\gamma(z)$ is bounded and hence admits a continuous extension onto $\mathcal{H}$. Furthermore, one has

$$
\gamma(z)^{*}: \mathfrak{H} \rightarrow \mathcal{H}, \quad f \mapsto \gamma(z)^{*} f=\Gamma_{1}\left(A_{0}-\bar{z} I_{\mathfrak{H}}\right)^{-1} f, \quad z \in \rho\left(A_{0}\right) .
$$


The values of the Weyl function $M(z), z \in \rho\left(A_{0}\right)$ are operators in $\mathcal{H}$ defined on $\operatorname{ran}\left(\Gamma_{0}\right)$ and mapping into ran $\left(\Gamma_{1}\right)$. The analogs of (2.6) and (2.7), and various other useful and important properties of the Weyl function can be found in $[5,6]$. In particular,

$$
M(z) \subseteq M(\bar{z})^{*}, \quad z \in \rho\left(A_{0}\right)
$$

and hence the operators $M(z), z \in \rho\left(A_{0}\right)$, are closable operators in $\mathcal{H}$. We point out that the operators $M(z), z \in \rho\left(A_{0}\right)$, and their closures are generally unbounded.

\section{The coupling model}

In this section we discuss the coupling issue mentioned in (1.15)-(1.17) from a purely abstract point of view.

Let $S_{+}$and $S_{-}$be densely defined closed symmetric operators in the separable Hilbert spaces $\mathfrak{H}_{+}$and $\mathfrak{H}_{-}$, respectively, and assume that the defect indices of $S_{+}$and $S_{-}$satisfy

$$
n_{+}\left(S_{+}\right)=n_{-}\left(S_{+}\right)=n_{+}\left(S_{-}\right)=n_{-}\left(S_{-}\right)=\infty .
$$

The case of finite defect numbers can be treated with the help of ordinary boundary triples in an efficient way and will not be discussed here (cf. [14]).

Let $T_{+}$and $T_{-}$be such that $\bar{T}_{+}=S_{+}^{*}$ and $\bar{T}_{-}=S_{-}^{*}$, and assume that $\left\{\mathcal{H}, \Gamma_{0}^{+}, \Gamma_{1}^{+}\right\}$ and $\left\{\mathcal{H}, \Gamma_{0}^{-}, \Gamma_{1}^{-}\right\}$are quasi boundary triples for $T_{+} \subseteq S_{+}^{*}$ and $T_{-} \subseteq S_{-}^{*}$, respectively. The corresponding $\gamma$-fields and Weyl functions are denoted by $\gamma_{+}$and $\gamma_{-}$, and $M_{+}$ and $M_{-}$, respectively. Furthermore, let

$$
A_{0,+}=T_{+} \uparrow \operatorname{ker}\left(\Gamma_{0}^{+}\right), \quad A_{0,-}=T_{-} \uparrow \operatorname{ker}\left(\Gamma_{0}^{-}\right)
$$

It is important to note that the identities

$$
\gamma_{+}(z)^{*}=\Gamma_{1}^{+}\left(A_{0,+}-\bar{z}\right)^{-1}, \quad \gamma_{-}(z)^{*}=\Gamma_{1}^{-}\left(A_{0,-}-\bar{z}\right)^{-1}
$$

hold for all $z \in \rho\left(A_{0,+}\right)$ and $z \in \rho\left(A_{0,-}\right)$, respectively [cf. (2.8)]. In the following consider the operators

$$
S=\left(\begin{array}{cc}
S_{+} & 0 \\
0 & S_{-}
\end{array}\right), \quad T=\left(\begin{array}{cc}
T_{+} & 0 \\
0 & T_{-}
\end{array}\right), \quad S^{*}=\left(\begin{array}{cc}
S_{+}^{*} & 0 \\
0 & S_{-}^{*}
\end{array}\right),
$$

in the Hilbert space $\mathfrak{H}=\mathfrak{H}_{+} \oplus \mathfrak{H}_{-}$. It is clear that $S$ is a closed, densely defined, symmetric operator in $\mathfrak{H}$ with equal infinite defect numbers,

$$
n_{+}(S)=n_{-}(S)=\infty
$$

and that

$$
\bar{T}=S^{*} .
$$


The elements $f$ in the domain of $S, T$ and $S^{*}$ will be written as two component vectors of the form $f=\left(f_{+}, f_{-}\right)^{\top}$, where $f_{ \pm}$belongs to the domain of $S_{ \pm}, T_{ \pm}$and $S_{ \pm}^{*}$, respectively. It is easy to see that $\left\{\mathcal{H} \oplus \mathcal{H}, \Gamma_{0}, \Gamma_{1}\right\}$, where

$$
\Gamma_{0} f=\left(\begin{array}{l}
\Gamma_{0}^{+} f_{+} \\
\Gamma_{0}^{-} f_{-}
\end{array}\right), \quad \Gamma_{1} f=\left(\begin{array}{l}
\Gamma_{1}^{+} f_{+} \\
\Gamma_{1}^{-} f_{-}
\end{array}\right), \quad f \in \operatorname{dom}(T),
$$

is a quasi boundary triple for $T \subseteq S^{*}$ such that

$$
A_{0}=T \uparrow \operatorname{ker}\left(\Gamma_{0}\right)=\left(\begin{array}{cc}
T_{+} \uparrow \operatorname{ker}\left(\Gamma_{0}^{+}\right) & 0 \\
0 & T_{-} \uparrow \operatorname{ker}\left(\Gamma_{0}^{-}\right)
\end{array}\right)=\left(\begin{array}{cc}
A_{0,+} & 0 \\
0 & A_{0,-}
\end{array}\right) .
$$

One notes that $\rho\left(A_{0}\right)=\rho\left(A_{0,+}\right) \cap \rho\left(A_{0,-}\right)$. The $\gamma$-field $\gamma$ and Weyl function $M$ corresponding to the quasi boundary triple $\left\{\mathcal{H} \oplus \mathcal{H}, \Gamma_{0}, \Gamma_{1}\right\}$ are given by

$$
\begin{gathered}
\gamma(z)=\left(\begin{array}{cc}
\gamma_{+}(z) & 0 \\
0 & \gamma_{-}(z)
\end{array}\right), \quad z \in \rho\left(A_{0}\right), \\
M(z)=\left(\begin{array}{cc}
M_{+}(z) & 0 \\
0 & M_{-}(z)
\end{array}\right), \quad z \in \rho\left(A_{0}\right),
\end{gathered}
$$

and (3.3) implies

$$
\gamma(z)^{*} f=\left(\begin{array}{l}
\Gamma_{1}^{+}\left(A_{0,+}-\bar{z}\right)^{-1} f_{+} \\
\Gamma_{1}^{-}\left(A_{0,-}-\bar{z}\right)^{-1} f_{-}
\end{array}\right), \quad f=\left(f_{+}, f_{-}\right)^{\top} \in \mathfrak{H} .
$$

The next result, Theorem 3.1, can be viewed as an abstract analogue of the coupling of differential operators, where $\Gamma_{0}^{ \pm}$are Dirichlet trace operators and $\Gamma_{1}^{ \pm}$are Neumann trace operators acting on different domains (cf. Section 5 for more details). We also note that in the following the operators $M_{+}(z)+M_{-}(z)$ in $\mathcal{H}$ are assumed to be defined on $\operatorname{dom}\left(M_{+}(z)\right) \cap \operatorname{dom}\left(M_{-}(z)\right)$.

Theorem 3.1 Let $\left\{\mathcal{H}, \Gamma_{0}^{+}, \Gamma_{1}^{+}\right\}$and $\left\{\mathcal{H}, \Gamma_{0}^{-}, \Gamma_{1}^{-}\right\}$be quasi boundary triples for $T_{+} \subseteq$ $S_{+}^{*}$ and $T_{-} \subseteq S_{-}^{*}$ with Weyl functions $M_{ \pm}$and define

$$
A:=T \nmid\left\{f=\left(f_{+}, f_{-}\right)^{\top} \in \operatorname{dom}(T) \mid \Gamma_{0}^{+} f_{+}=\Gamma_{0}^{-} f_{-}, \Gamma_{1}^{+} f_{+}=-\Gamma_{1}^{-} f_{-}\right\} .
$$

Then the following assertions (i)-(iii) hold:

(i) A is a symmetric operator in $\mathfrak{H}$.

(ii) $z \in \rho\left(A_{0}\right)$ is an eigenvalue of $A$ if and only if

$$
\operatorname{ker}\left(M_{+}(z)+M_{-}(z)\right) \neq\{0\} .
$$

(iii) A is a self-adjoint operator in $\mathfrak{H}$ if and only if

$$
\operatorname{ran}\left(\Gamma_{1}^{ \pm}\left\lceil\operatorname{dom}\left(A_{0, \pm}\right)\right) \subset \operatorname{ran}\left(M_{+}(z)+M_{-}(z)\right)\right.
$$

holds for some (and hence for all ) $z \in \mathbb{C}_{+}$and some (and hence for all) $z \in \mathbb{C}_{-}$. 
If $A$ is a self-adjoint operator in $\mathfrak{H}$ then for all $z \in \rho(A) \cap \rho\left(A_{0}\right)$ the resolvent of $A$ is given in terms of a Krein-type resolvent formula by

$$
\left(A-z I_{\mathfrak{H}}\right)^{-1}=\left(A_{0}-z I_{\mathfrak{H}}\right)^{-1}+\gamma(z) \Theta(z) \gamma(\bar{z})^{*},
$$

where

$$
\Theta(z):=-\left(\begin{array}{cc}
\left(M_{+}(z)+M_{-}(z)\right)^{-1} & \left(M_{+}(z)+M_{-}(z)\right)^{-1} \\
\left(M_{+}(z)+M_{-}(z)\right)^{-1} & \left(M_{+}(z)+M_{-}(z)\right)^{-1}
\end{array}\right) .
$$

Remark 3.2 The perturbation term $\gamma(z) \Theta(z) \gamma(\bar{z})^{*}$ in the right-hand side of (3.15) may also be written in the form

$$
-\left(\begin{array}{l}
\gamma_{+}(z) \\
\gamma_{-}(z)
\end{array}\right)\left(M_{+}(z)+M_{-}(z)\right)^{-1}\left(\gamma_{+}(\bar{z})^{*}, \gamma_{-}(\bar{z})^{*}\right)
$$

Proof of Theorem 3.1 (i) In order to show that $A$ is a symmetric operator in $\mathfrak{H}$ let $f=\left(f_{+}, f_{-}\right)^{\top}$ and $g=\left(g_{+}, g_{-}\right)^{\top}$ be in $\operatorname{dom}(A)$. Making use of the abstract boundary conditions for $f, g \in \operatorname{dom}(A)$, a straightforward computation using the abstract Green identity (2.1) shows that

$$
\begin{aligned}
(A f, g)_{\mathfrak{H}}-(f, A g)_{\mathfrak{H}} \\
=\left(T_{+} f_{+}, g_{+}\right)_{\mathfrak{H}_{+}}-\left(f_{+}, T_{+} g_{+}\right)_{\mathfrak{H}_{+}}+\left(T_{-} f_{-}, g_{-}\right)_{\mathfrak{H}_{-}}-\left(f_{-}, T_{-} g_{-}\right)_{\mathfrak{H}_{-}} \\
=\left(\Gamma_{1}^{+} f_{+}, \Gamma_{0}^{+} g_{+}\right)_{\mathcal{H}}-\left(\Gamma_{0}^{+} f_{+}, \Gamma_{1}^{+} g_{+}\right)_{\mathcal{H}} \\
\quad+\left(\Gamma_{1}^{-} f_{-}, \Gamma_{0}^{-} g_{-}\right)_{\mathcal{H}}-\left(\Gamma_{0}^{-} f_{-}, \Gamma_{1}^{-} g_{-}\right)_{\mathcal{H}} \\
=\left(\Gamma_{1}^{+} f_{+}+\Gamma_{1}^{-} f_{-}, \Gamma_{0}^{+} g_{+}\right)_{\mathcal{H}}-\left(\Gamma_{0}^{+} f_{+}, \Gamma_{1}^{+} g_{+}+\Gamma_{1}^{-} g_{-}\right)_{\mathcal{H}} \\
=0
\end{aligned}
$$

hence $A$ is symmetric.

(ii) Let $z \in \rho\left(A_{0}\right)$ and assume that $z$ is an eigenvalue of $A$. Considering $f \in \operatorname{ker}(A-$ $\left.z I_{\mathfrak{H}}\right), f \neq 0$, one observes that $\Gamma_{0} f \neq 0$ as otherwise $f \in \operatorname{dom}\left(A_{0}\right)$ would be an eigenfunction of $A_{0}$ at $z$. Clearly $f \in \operatorname{ker}\left(T-z I_{\mathfrak{H}}\right)$ and hence $M(z) \Gamma_{0} f=\Gamma_{1} f$. For $f=\left(f_{+}, f_{-}\right)^{\top}$ one then obtains

$$
M_{+}(z) \Gamma_{0}^{+} f_{+}=\Gamma_{1}^{+} f_{+} \text {and } M_{-}(z) \Gamma_{0}^{-} f_{-}=\Gamma_{1}^{-} f_{-} .
$$

Since $f \in \operatorname{dom}(A)$ satisfies $\Gamma_{0}^{+} f_{+}=\Gamma_{0}^{-} f_{-}$and $\Gamma_{1}^{+} f_{+}+\Gamma_{1}^{-} f_{-}=0$, one concludes

$$
\left(M_{+}(z)+M_{-}(z)\right) \Gamma_{0}^{ \pm} f_{ \pm}=M_{+}(z) \Gamma_{0}^{+} f_{+}+M_{-}(z) \Gamma_{0}^{-} f_{-}=\Gamma_{1}^{+} f_{+}+\Gamma_{1}^{-} f_{-}=0
$$

As a consequence of $\Gamma_{0}^{ \pm} f_{ \pm} \neq 0$, it follows that $\operatorname{ker}\left(M_{+}(z)+M_{-}(z)\right) \neq\{0\}$. 
Conversely, assume that $\varphi \in \operatorname{ker}\left(M_{+}(z)+M_{-}(z)\right), \varphi \neq 0$, for some $z \in \rho\left(A_{0}\right)$. One notes that

$$
M_{+}(z) \varphi=-M_{-}(z) \varphi \text { and } \varphi \in \operatorname{ran}\left(\Gamma_{0}^{+}\right) \cap \operatorname{ran}\left(\Gamma_{0}^{-}\right) .
$$

Hence there exist $f_{+} \in \operatorname{ker}\left(T_{+}-z I_{\mathfrak{H}_{+}}\right)$and $f_{-} \in \operatorname{ker}\left(T_{-}-z I_{\mathfrak{H}_{-}}\right)$such that

$$
\Gamma_{0}^{+} f_{+}=\varphi=\Gamma_{0}^{-} f_{-} .
$$

From the definition of $M_{+}$and $M_{-}$, and (3.21)-(3.22) one concludes that

$$
\Gamma_{1}^{+} f_{+}=M_{+}(z) \Gamma_{0}^{+} f_{+}=-M_{-}(z) \Gamma_{0}^{-} f_{-}=-\Gamma_{1}^{-} f_{-},
$$

and hence (3.22) and (3.23) show that $f=\left(f_{+}, f_{-}\right)^{\top} \in \operatorname{ker}\left(T-z I_{\mathfrak{H}}\right)$ satisfies both abstract boundary conditions for elements in $\operatorname{dom}(A)$. Thus, $f \in \operatorname{ker}\left(A-z I_{\mathfrak{H}}\right)$. (iii) First, assume that $A$ is a self-adjoint operator in $\mathfrak{H}$, fix $z \in \mathbb{C} \backslash \mathbb{R}$, and let

$$
\varphi_{+} \in \operatorname{ran}\left(\Gamma_{1}^{+} \uparrow \operatorname{dom}\left(A_{0,+}\right)\right) \text {. }
$$

Then there exists $f_{+} \in \mathfrak{H}_{+}$such that

$$
\varphi_{+}=\Gamma_{1}^{+}\left(A_{0,+}-z I_{\mathfrak{H}_{+}}\right)^{-1} f_{+}=\gamma_{+}(\bar{z})^{*} f_{+},
$$

where the last identity follows from (3.3). Next, consider $f=\left(f_{+}, 0\right)^{\top} \in \mathfrak{H}$, set

$$
h:=\left(A-z I_{\mathfrak{H}}\right)^{-1} f-\left(A_{0}-z I_{\mathfrak{H}}\right)^{-1} f \in \operatorname{ker}\left(T-z I_{\mathfrak{H}}\right),
$$

and

$$
k:=\left(A-z I_{\mathfrak{H}}\right)^{-1} f \in \operatorname{dom}(A) .
$$

Then one has $\Gamma_{0} h=\Gamma_{0} k$ and $\Gamma_{1} h=\Gamma_{1} k-\gamma(\bar{z})^{*} f$ and hence

$$
\gamma(\bar{z})^{*} f=\Gamma_{1} k-\Gamma_{1} h=\Gamma_{1} k-M(z) \Gamma_{0} h=\Gamma_{1} k-M(z) \Gamma_{0} k .
$$

Making use of (3.25) and $f_{-}=0$ this reads componentwise as

$$
\begin{aligned}
\varphi_{+} & =\gamma_{+}(\bar{z})^{*} f_{+}=\Gamma_{1}^{+} k_{+}-M_{+}(z) \Gamma_{0}^{+} k_{+}, \\
0 & =\gamma_{-}(\bar{z})^{*} f_{-}=\Gamma_{1}^{-} k_{-}-M_{-}(z) \Gamma_{0}^{-} k_{-}
\end{aligned}
$$

[cf. (3.11)]. Summing up these two equations and taking into account that $k \in \operatorname{dom}(A)$ satisfies $\Gamma_{1}^{+} k_{+}+\Gamma_{1}^{-} k_{-}=0$ and $\Gamma_{0}^{+} k_{+}=\Gamma_{0}^{-} k_{-}$, one finds

$$
\varphi_{+}=\gamma_{+}(\bar{z})^{*} f_{+}=-\left(M_{+}(z)+M_{-}(z)\right) \Gamma_{0}^{+} k_{+} .
$$

Hence, the inclusion

$$
\operatorname{ran}\left(\Gamma_{1}^{+}\left\lceil\operatorname{dom}\left(A_{0,+}\right)\right) \subseteq \operatorname{ran}\left(M_{+}(z)+M_{-}(z)\right)\right.
$$


holds for any $z \in \mathbb{C} \backslash \mathbb{R}$. In the same way as above one also shows the inclusion

$$
\operatorname{ran}\left(\Gamma_{1}^{-} \uparrow \operatorname{dom}\left(A_{0,-}\right)\right) \subseteq \operatorname{ran}\left(M_{+}(z)+M_{-}(z)\right)
$$

Next, we will prove the converse. Assume that there exist $z_{ \pm} \in \mathbb{C}_{ \pm}$such that

$$
\operatorname{ran}\left(\Gamma_{1}^{ \pm}\left\lceil\operatorname{dom}\left(A_{0, \pm}\right)\right) \subseteq \operatorname{ran}\left(M_{+}(z)+M_{-}(z)\right)\right.
$$

holds for $z \in\left\{z_{ \pm}\right\}$. We have to prove that the operator $A$ is self-adjoint in $\mathfrak{H}$. Along the way we will also show that the resolvent formula holds at the point $z$. Note first that $A$ is symmetric by item (i) and hence all eigenvalues of $A$ are real. In particular, $z$ is not an eigenvalue of $A$ and according to item (ii), the operator $M_{+}(z)+M_{-}(z)$ is injective. Let $f=\left(f_{+}, f_{-}\right)^{\top} \in \mathfrak{H}$ and note that [cf. (3.11)]

$$
\gamma(\bar{z})^{*} f=\left(\begin{array}{l}
\Gamma_{1}^{+}\left(A_{0,+}-z I_{\mathfrak{H}_{+}}\right)^{-1} f_{+} \\
\Gamma_{1}^{-}\left(A_{0,-}-z I_{\mathfrak{H}_{-}}\right)^{-1} f_{-}
\end{array}\right)
$$

and that

$$
\Gamma_{1}^{ \pm}\left(A_{0, \pm}-z I_{\mathfrak{H}_{ \pm}}\right)^{-1} f_{ \pm} \in \operatorname{ran}\left(M_{+}(z)+M_{-}(z)\right)=\operatorname{dom}\left(\left(M_{+}(z)+M_{-}(z)\right)^{-1}\right)
$$

by assumption. Now consider the element

$g:=\left(A_{0}-z I_{\mathfrak{H}}\right)^{-1} f-\gamma(z)\left(\begin{array}{c}\left(M_{+}(z)+M_{-}(z)\right)^{-1}\left(M_{+}(z)+M_{-}(z)\right)^{-1} \\ \left(M_{+}(z)+M_{-}(z)\right)^{-1}\left(M_{+}(z)+M_{-}(z)\right)^{-1}\end{array}\right) \gamma(\bar{z})^{*} f$,

which is well-defined by the above considerations and the fact that

$$
\operatorname{dom}(\gamma(z))=\operatorname{dom}\left(\left(\begin{array}{cc}
\gamma_{+}(z) & 0 \\
0 & \gamma_{-}(z)
\end{array}\right)\right)=\operatorname{ran}\left(\Gamma_{0}^{+}\right) \times \operatorname{ran}\left(\Gamma_{0}^{-}\right)
$$

and

$$
\operatorname{ran}\left(\left(M_{+}(z)+M_{-}(z)\right)^{-1}\right)=\operatorname{dom}\left(M_{+}(z)+M_{-}(z)\right)=\operatorname{ran}\left(\Gamma_{0}^{+}\right) \cap \operatorname{ran}\left(\Gamma_{0}^{-}\right) .
$$

Since $\left(A_{0}-z I_{\mathfrak{H}}\right)^{-1} f \in \operatorname{dom}\left(A_{0}\right) \subset \operatorname{dom}(T)$ and $\operatorname{ran}(\gamma(z))=\operatorname{ker}\left(T-z I_{\mathfrak{H}}\right) \subset$ $\operatorname{dom}(T)$, it is clear that $g \in \operatorname{dom}(T)$. Next, it will be shown that $g=\left(g_{+}, g_{-}\right)^{\top}$ satisfies the boundary conditions

$$
\Gamma_{0}^{+} g_{+}=\Gamma_{0}^{-} g_{-} \text {and } \Gamma_{1}^{+} g_{+}=-\Gamma_{1}^{-} g_{-} .
$$

Due to

$$
\left(A_{0}-z I_{\mathfrak{H}}\right)^{-1}=\left(\begin{array}{cc}
\left(A_{0,+}-z I_{\mathfrak{H}_{+}}\right)^{-1} & 0 \\
0 & \left(A_{0,-}-z I_{\mathfrak{H}_{-}}\right)^{-1}
\end{array}\right)
$$


and the special form of $\gamma(z)$ and $\gamma(\bar{z})^{*}$, one infers that

$$
g_{+}=\left(A_{0,+}-z I_{\mathfrak{H}_{+}}\right)^{-1} f_{+}-\gamma_{+}(z)\left(M_{+}(z)+M_{-}(z)\right)^{-1}\left(\gamma_{+}(\bar{z})^{*} f_{+}+\gamma_{-}(\bar{z})^{*} f_{-}\right),
$$

and

$$
g_{-}=\left(A_{0,-}-z I_{\mathfrak{H}_{-}}\right)^{-1} f_{-}-\gamma_{-}(z)\left(M_{+}(z)+M_{-}(z)\right)^{-1}\left(\gamma_{+}(\bar{z})^{*} f_{+}+\gamma_{-}(\bar{z})^{*} f_{-}\right) .
$$

Since by definition, $A_{0, \pm}=T_{ \pm} \uparrow \operatorname{ker}\left(\Gamma_{0}^{ \pm}\right)$and $\gamma_{ \pm}(z)=\left(\Gamma_{0}^{ \pm} \uparrow \operatorname{ker}\left(T_{ \pm}-z I_{\mathfrak{H}_{ \pm}}\right)\right)^{-1}$, one obtains

$$
\begin{aligned}
& \Gamma_{0}^{+} g_{+}=-\left(M_{+}(z)+M_{-}(z)\right)^{-1}\left(\gamma_{+}(\bar{z})^{*} f_{+}+\gamma_{-}(\bar{z})^{*} f_{-}\right), \\
& \Gamma_{0}^{-} g_{-}=-\left(M_{+}(z)+M_{-}(z)\right)^{-1}\left(\gamma_{+}(\bar{z})^{*} f_{+}+\gamma_{-}(\bar{z})^{*} f_{-}\right),
\end{aligned}
$$

and hence the first condition in (3.39) is satisfied. Next, we make use of (3.3) and $M_{ \pm}(z)=\Gamma_{1}^{ \pm} \gamma_{ \pm}(z)$ and compute

$$
\begin{aligned}
& \Gamma_{1}^{+} g_{+}=\gamma_{+}(\bar{z})^{*} f_{+}-M_{+}(z)\left(M_{+}(z)+M_{-}(z)\right)^{-1}\left(\gamma_{+}(\bar{z})^{*} f_{+}+\gamma_{-}(\bar{z})^{*} f_{-}\right) \\
& \Gamma_{1}^{-} g_{-}=\gamma_{-}(\bar{z})^{*} f_{-}-M_{-}(z)\left(M_{+}(z)+M_{-}(z)\right)^{-1}\left(\gamma_{+}(\bar{z})^{*} f_{+}+\gamma_{-}(\bar{z})^{*} f_{-}\right)
\end{aligned}
$$

It follows that $\Gamma_{1}^{+} g_{+}+\Gamma_{1}^{-} g_{-}=0$. Hence also the second boundary condition in (3.39) is satisfied. Therefore, $g \in \operatorname{dom}(A)$, and when applying $\left(A-z I_{\mathfrak{H}}\right)$ to $g$ it follows from the particular form of $g$ and $\operatorname{ran}(\gamma(z)) \subseteq \operatorname{ker}\left(T-z I_{\mathfrak{H}}\right)$ that

$$
\left(A-z I_{\mathfrak{H}}\right) g=\left(T-z I_{\mathfrak{H}}\right)\left(A_{0}-z I_{\mathfrak{H}}\right)^{-1} f=f .
$$

Furthermore, as $A$ is symmetric, $z$ is not an eigenvalue of $A$ and one concludes that

$$
\left(A-z I_{\mathfrak{H}}\right)^{-1} f=g .
$$

Since $f \in \mathfrak{H}$ was chosen arbitrary it follows that $\left(A-z I_{\mathfrak{H}}\right)^{-1}$ is an everywhere defined operator in $\mathfrak{H}$. By our assumptions this is true for $z=\left\{z_{ \pm}\right\}$. Hence it follows that $A$ is self-adjoint and that the resolvent of $A$ at the point $z$ has the asserted form, proving assertion (iii).

It remains to show that the resolvent of $A$ is of the form as stated in the theorem for all $z \in \rho(A) \cap \rho\left(A_{0}\right)$. For this we remark that

$$
\gamma_{+}(\bar{z})^{*} f_{+}+\gamma_{-}(\bar{z})^{*} f_{-} \in \operatorname{ran}\left(M_{+}(z)+M_{-}(z)\right)
$$

holds for all $z \in \rho(A) \cap \rho\left(A_{0}\right)$ and $f=\left(f_{+}, f_{-}\right)^{\top} \in \mathfrak{H}$; this follows essentially from the first part of the proof of item (iii) (which remains valid for points in $\rho(A) \cap \rho\left(A_{0}\right)$ ). Based on (3.49) and the fact that $\operatorname{ker}\left(M_{+}(z)+M_{-}(z)\right)=\{0\}$ for all $z \in \rho(A) \cap \rho\left(A_{0}\right)$ 
by assertion (ii), it can be shown in the same way as in the second part of the proof of item (iii) that the resolvent of $A$ has the asserted form. This completes the proof of Theorem 3.1.

The next step is to derive a slightly modified formula for the resolvent of $A$ in Theorem 3.1 where the resolvent of $A_{0}$ is replaced by the resolvent of the operator

$$
\left(\begin{array}{cc}
A_{0,+} & 0 \\
0 & A_{1,-}
\end{array}\right)
$$

where

$$
A_{1,-}=T_{-} \uparrow \operatorname{ker}\left(\Gamma_{1}^{-}\right)
$$

is assumed to be a self-adjoint operator in $\mathfrak{H}_{-}$. We recall that in the context of quasi boundary triples, the extension $A_{1,-}$ of $S_{-}$corresponding to $\operatorname{ker}\left(\Gamma_{1}^{-}\right)$is always symmetric, but generally not self-adjoint. The resolvent formula in the next theorem is essentially a consequence of Theorem 3.1 and a formula relating the resolvent of $A_{0,-}$ with the resolvent of $A_{1,-}$.

Theorem 3.3 Let $\left\{\mathcal{H}, \Gamma_{0}^{+}, \Gamma_{1}^{+}\right\}$and $\left\{\mathcal{H}, \Gamma_{0}^{-}, \Gamma_{1}^{-}\right\}$be quasi boundary triples for $T_{+} \subseteq$ $S_{+}^{*}$ and $T_{-} \subseteq S_{-}^{*}$ with Weyl functions $M_{ \pm}$as in Theorem 3.1. Assume, in addition, that $A_{1,-}=T_{-} \uparrow \operatorname{ker}\left(\Gamma_{1}^{-}\right)$is self-adjoint in $\mathfrak{H}_{-}$, and let $A$ in Theorem 3.1 be self-adjoint in $\mathfrak{H}$. Then for all $z \in \rho(A) \cap \rho\left(A_{0,+}\right) \cap \rho\left(A_{0,-}\right) \cap \rho\left(A_{1,-}\right)$ the resolvent of $A$ is given by

$$
\left(A-z I_{\mathfrak{H}}\right)^{-1}=\left(\left(\begin{array}{cc}
A_{0,+} & 0 \\
0 & A_{1,-}
\end{array}\right)-z I_{\mathfrak{H}}\right)^{-1}+\widehat{\gamma}(z) \Sigma(z) \widehat{\gamma}(\bar{z})^{*},
$$

where

$$
\widehat{\gamma}(z)=\left(\begin{array}{cc}
\gamma_{+}(z) & 0 \\
0 & \gamma_{-}(z) M_{-}(z)^{-1}
\end{array}\right), \quad \Sigma(z)=-\left(\begin{array}{cc}
M_{+}(z) & I_{\mathcal{H}} \\
I_{\mathcal{H}} & -M_{-}(z)^{-1}
\end{array}\right)^{-1} .
$$

Proof Since $A_{1,-}=T_{-} \uparrow \operatorname{ker}\left(\Gamma_{1}^{-}\right)$is self-adjoint in $\mathfrak{H}_{-}$it follows from [6, Theorem 6.16] that $M_{-}(z)$ is injective for all $z \in \rho\left(A_{0,-}\right) \cap \rho\left(A_{1,-}\right)$ and the resolvents of $A_{0,-}$ and $A_{1,-}$ are related via

$$
\left(A_{0,-}-z I_{\mathfrak{H}_{-}}\right)^{-1}=\left(A_{1,-}-z I_{\mathfrak{H}_{-}}\right)^{-1}+\gamma_{-}(z) M_{-}(z)^{-1} \gamma_{-}(\bar{z})^{*}
$$

for all $z \in \rho\left(A_{0,-}\right) \cap \rho\left(A_{1,-}\right)$. Making use of (3.8) and inserting (3.54) in (3.15)(3.16) one obtains

$$
\left(A-z I_{\mathfrak{H}}\right)^{-1}=\left(\left(\begin{array}{cc}
A_{0,+} & 0 \\
0 & A_{1,-}
\end{array}\right)-z I_{\mathfrak{H}}\right)^{-1}+\widehat{\gamma}(z) \Psi(z) \widehat{\gamma}(\bar{z})^{*}
$$

where

$$
\Psi(z)=\left(\begin{array}{cc}
I_{\mathcal{H}} & 0 \\
0 & M_{-}(z)
\end{array}\right)\left[\left(\begin{array}{ll}
0 & 0 \\
0 & M_{-}(z)^{-1}
\end{array}\right)+\Theta(z)\right]\left(\begin{array}{cc}
I_{\mathcal{H}} & 0 \\
0 & M_{-}(z)
\end{array}\right)
$$


and we have used that

$$
\left(\gamma_{-}(\bar{z}) M_{-}(\bar{z})^{-1}\right)^{*}=\left(M_{-}(\bar{z})^{*}\right)^{-1} \gamma_{-}(\bar{z})^{*}=M_{-}(z)^{-1} \gamma_{-}(\bar{z})^{*}
$$

Here the first equality holds since $\gamma_{-}(\bar{z})^{*}$ is everywhere defined and bounded, and the second equality is valid since $M_{-}(z)^{-1} \subset\left(M_{-}(\bar{z})^{*}\right)^{-1}$ and

$$
\operatorname{ran}\left(\gamma_{-}(\bar{z})^{*}\right) \subset \operatorname{ran}\left(\Gamma_{1}\right)=\operatorname{dom}\left(M_{-}(z)^{-1}\right), \quad z \in \rho\left(A_{0,-}\right) \cap \rho\left(A_{1,-}\right) .
$$

The block operator matrix $\Psi(z)$ in $\mathcal{H}^{2}$ has the form

$$
\Psi(z)=\left(\begin{array}{cc}
-\left(M_{+}(z)+M_{-}(z)\right)^{-1} & -\left(M_{+}(z)+M_{-}(z)\right)^{-1} M_{-}(z) \\
-M_{-}(z)\left(M_{+}(z)+M_{-}(z)\right)^{-1} & M_{+}(z)\left(M_{+}(z)+M_{-}(z)\right)^{-1} M_{-}(z)
\end{array}\right)
$$

and from this representation one infers that $\Psi(z)=\Sigma(z)$ for all $z \in \rho(A) \cap \rho\left(A_{0,+}\right) \cap$ $\rho\left(A_{0,-}\right) \cap \rho\left(A_{1,-}\right)$.

In the case where $\left\{\mathcal{H}, \Gamma_{0}^{+}, \Gamma_{1}^{+}\right\}$is an ordinary boundary triple, Eq. (3.52) was proved in [16, Eq. (6.7)] (cf. also [14]).

\section{The third Green identity}

This section is devoted to an abstract version of the third Green identity [cf. (1.19) for the concrete example that motivated these investigations].

Let $T$ be given by (3.4) and let $\left\{\mathcal{H}, \Gamma_{0}^{+}, \Gamma_{1}^{+}\right\}$and $\left\{\mathcal{H}, \Gamma_{0}^{-}, \Gamma_{1}^{-}\right\}$be quasi boundary triples for $T_{+} \subseteq S_{+}^{*}$ and $T_{-} \subseteq S_{-}^{*}$, respectively. We will investigate the operator

$$
A=T\left\lceil\left\{f=\left(f_{+}, f_{-}\right)^{\top} \in \operatorname{dom}(T) \mid \Gamma_{0}^{+} f_{+}=\Gamma_{0}^{-} f_{-}, \Gamma_{1}^{+} f_{+}=-\Gamma_{1}^{-} f_{-}\right\},\right.
$$

which corresponds to the coupling of the quasi-boundary triples $\left\{\mathcal{H}, \Gamma_{0}^{+}, \Gamma_{1}^{+}\right\}$and $\left\{\mathcal{H}, \Gamma_{0}^{-}, \Gamma_{1}^{-}\right\}$in Theorem 3.1. One recalls that $A$ is a symmetric operator in the Hilbert space $\mathfrak{H}=\mathfrak{H}_{+} \oplus \mathfrak{H}_{-}$. From now on we shall assume that the following hypothesis is satisfied [cf. Theorem 3.1(iii)].

Hypothesis 4.1 The operator A in (4.1) is self-adjoint in the Hilbert space $\mathfrak{H}$.

In the following let $\mathfrak{H}_{2}:=\operatorname{dom}(A)$ be the Hilbert space with inner product

$$
(f, g)_{\mathfrak{H}_{2}}:=(A f, A g)_{\mathfrak{H}}+(f, g)_{\mathfrak{H}}, \quad f, g \in \operatorname{dom}(A)
$$

and let $\mathfrak{H}_{-2}$ be the space of anti-linear continuous functionals on $\mathfrak{H}_{2}$ with the pairing denoted by

$$
\mathfrak{H}_{-2}\langle h, g\rangle_{\mathfrak{H}_{2}}, \quad g \in \mathfrak{H}_{2}, \quad h \in \mathfrak{H}_{-2} .
$$

Since for every $f \in \mathfrak{H}$ the functional $(f, \cdot)_{\mathfrak{H}}$ is bounded on $\mathfrak{H}_{2}$, the space $\mathfrak{H}$ embeds densely into the space $\mathfrak{H}_{-2}$ in such a way that (cf. [10, Section 1])

$$
\mathfrak{H}_{-2}\langle f, g\rangle_{\mathfrak{H}_{2}}=(f, g)_{\mathfrak{H}}, \quad f \in \mathfrak{H}, \quad g \in \mathfrak{H}_{2},
$$


leading to a Gelfand triple of the form

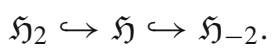

Let $\mathcal{A}$ be the dual operator to $A$ determined by

$$
\mathfrak{H}_{-2}\langle\mathcal{A} f, g\rangle_{\mathfrak{H}_{2}}:=(f, A g)_{\mathfrak{H}}, \quad f \in \mathfrak{H}, \quad g \in \mathfrak{H}_{2}
$$

Since $A \in \mathcal{B}\left(\mathfrak{H}_{2}, \mathfrak{H}\right)$, also $\mathcal{A} \in \mathcal{B}\left(\mathfrak{H}_{1}, \mathfrak{H}_{-2}\right)$.

Next, define the map $\Upsilon$ on $\mathfrak{H}_{2}=\operatorname{dom}(A)$ as the restriction of $\left(\Gamma_{0}^{+}, \Gamma_{1}^{+}\right)^{\top}$,

$$
\Upsilon: \mathfrak{H}_{2} \rightarrow \mathcal{H}^{2}, \quad f \mapsto \Upsilon f=\left(\begin{array}{c}
\Upsilon_{0} f \\
\Upsilon_{1} f
\end{array}\right):=\left(\begin{array}{c}
\Gamma_{0}^{+} f_{+} \\
\Gamma_{1}^{+} f_{+}
\end{array}\right)=\left(\begin{array}{c}
\Gamma_{0}^{-} f_{-} \\
-\Gamma_{1}^{-} f_{-}
\end{array}\right) .
$$

Here the last equality follows from the abstract boundary conditions in (4.1) for all $f \in \operatorname{dom}(A)$.

Lemma 4.2 Assume Hypothesis 4.1 and suppose that $A$ in (4.1) corresponds to the coupling of the quasi-boundary triples $\left\{\mathcal{H}, \Gamma_{0}^{+}, \Gamma_{1}^{+}\right\}$and $\left\{\mathcal{H}, \Gamma_{0}^{-}, \Gamma_{1}^{-}\right\}$for $T_{+} \subseteq S_{+}^{*}$ and $T_{-} \subseteq S_{-}^{*}$, respectively. Then the operators $\Upsilon_{0}, \Upsilon_{1}: \mathfrak{H}_{2} \rightarrow \mathcal{H}$ in (4.7) are bounded.

Proof We start by showing that the map $\Upsilon$ is closable from $\mathfrak{H}_{2}$ to $\mathcal{H}^{2}$. Assume that

$$
\lim _{n \rightarrow \infty} f_{n}=0, \quad \lim _{n \rightarrow \infty} A f_{n}=0, \quad \lim _{n \rightarrow \infty} \Upsilon_{0} f_{n}=\varphi, \quad \lim _{n \rightarrow \infty} \Upsilon_{1} f_{n}=\psi
$$

for some $\varphi, \psi \in \mathcal{H}$. Then by (4.7),

$$
\lim _{n \rightarrow \infty} f_{n,+}=0, \quad \lim _{n \rightarrow \infty} T_{+} f_{n,+}=0, \quad \lim _{n \rightarrow \infty} \Gamma_{0}^{+} f_{n,+}=\varphi, \quad \lim _{n \rightarrow \infty} \Gamma_{1}^{+} f_{n,+}=\psi .
$$

Since the map $\left(\Gamma_{0}^{+}, \Gamma_{1}^{+}\right)^{\top}: \operatorname{dom}(T) \rightarrow \mathcal{H}^{2}$ is closable by [5, Proposition 2.2] one concludes that $\varphi=\psi=0$ and hence the map $\Upsilon: \mathfrak{H}_{2} \rightarrow \mathcal{H}^{2}$ is closable. Since $\operatorname{dom}(\Upsilon)=\mathfrak{H}_{2}$, it follows that $\Upsilon$ is closed, and the closed graph theorem implies that $\Upsilon: \mathfrak{H}_{2} \rightarrow \mathcal{H}^{2}$ is bounded. Thus, also $\Upsilon_{0}, \Upsilon_{1}: \mathfrak{H}_{2} \rightarrow \mathcal{H}$ are bounded.

Let $\Upsilon_{j}^{*}: \mathcal{H} \rightarrow \mathfrak{H}_{-2}$ be the dual operator to $\Upsilon_{j}: \mathfrak{H}_{2} \rightarrow \mathcal{H}, j=0,1$, in (4.7) determined by

$$
\mathfrak{H}_{-2}\left\langle\Upsilon_{j}^{*} \varphi, g\right\rangle_{\mathfrak{H}_{2}}:=\left(\varphi, \Upsilon_{j} g\right)_{\mathcal{H}}, \quad \varphi \in \mathcal{H}, g \in \mathfrak{H}_{2}, j=0,1
$$

Since $\Upsilon_{j}$ are bounded operators from $\mathfrak{H}_{2}$ to $\mathcal{H}$ by Lemma 4.2, it is clear that $\Upsilon_{j}^{*}$, $j=1,2$, are bounded operators from $\mathcal{H}$ to $\mathfrak{H}_{-2}$.

Next we introduce an abstract analog of the single and double layer potential (cf. [29]). For this it will be assumed that there is an abstract fundamental solution operator for $\mathcal{A}$. 
Hypothesis 4.3 There exists a linear operator $\mathcal{G}: \mathfrak{H}_{-2} \rightarrow \mathfrak{H}$ such that

$$
\mathcal{G} \mathcal{A} f=f, \quad f \in \mathfrak{H} .
$$

Definition 4.4 Assume Hypotheses 4.1 and 4.3 and let $\Upsilon_{0}^{*}, \Upsilon_{1}^{*}: \mathcal{H} \rightarrow \mathfrak{H}_{-2}$ be defined by (4.10). The abstract single and double layer potentials are defined by

$$
\mathcal{S}:\left\{\begin{array}{l}
\mathcal{H} \rightarrow \mathfrak{H}, \\
\varphi \mapsto \mathcal{G} \Upsilon_{0}^{*} \varphi,
\end{array}\right.
$$

and

$$
\mathcal{D}:\left\{\begin{array}{l}
\mathcal{H} \rightarrow \mathfrak{H}, \\
\varphi \mapsto-\mathcal{G} \Upsilon_{1}^{*} \varphi,
\end{array}\right.
$$

respectively.

It is clear that the operators $\mathcal{S}$ and $\mathcal{D}$ are well-defined. In order to obtain an abstract third Green identity in the next theorem we will also use the following notations for the "jumps" of boundary values:

$$
\left[\Gamma_{0} f\right]:=\Gamma_{0}^{-} f_{-}-\Gamma_{0}^{+} f_{+}, \quad f=\left(f_{+}, f_{-}\right)^{\top} \in \operatorname{dom}(T),
$$

and

$$
\left[\Gamma_{1} f\right]:=\Gamma_{1}^{+} f_{+}+\Gamma_{1}^{-} f_{-}, \quad f=\left(f_{+}, f_{-}\right)^{\top} \in \operatorname{dom}(T) .
$$

One observes that the jump notations in (4.14)-(4.15) are compatible with the boundary conditions for elements in $\operatorname{dom}(A)$; we note that different signs are used in (4.15) since in the application in Sect. 5 the operators $\Gamma_{1}^{ \pm}$will be the normal derivatives with the normals having opposite direction.

Theorem 4.5 Assume Hypotheses 4.1 and 4.3 and suppose that A in (4.1) corresponds to the coupling of the quasi-boundary triples $\left\{\mathcal{H}, \Gamma_{0}^{+}, \Gamma_{1}^{+}\right\}$and $\left\{\mathcal{H}, \Gamma_{0}^{-}, \Gamma_{1}^{-}\right\}$for $T_{+} \subseteq$ $S_{+}^{*}$ and $T_{-} \subseteq S_{-}^{*}$, respectively. Let $\left[\Gamma_{0} \cdot\right],\left[\Gamma_{1} \cdot\right], \mathcal{S}$, and $\mathcal{D}$ be defined as above. Then

$$
f=\mathcal{G} T f+\mathcal{D}\left[\Gamma_{0} f\right]-\mathcal{S}\left[\Gamma_{1} f\right], \quad f \in \operatorname{dom}(T) .
$$

Proof Let $f=\left(f_{+}, f_{-}\right)^{\top} \in \operatorname{dom}(T)$ and $g=\left(g_{+}, g_{-}\right)^{\top} \in \operatorname{dom}(A)$. Then it follows from (4.6) and the abstract Green identity (2.1) that

$$
\begin{aligned}
\mathfrak{H}_{-2}\langle\mathcal{A} f, g\rangle_{\mathfrak{H}_{2}=} & (f, A g)_{\mathfrak{H}} \\
= & \left(f_{+}, T_{+} g_{+}\right)_{\mathfrak{H}_{+}}+\left(f_{-}, T_{-} g_{-}\right)_{\mathfrak{H}_{-}} \\
= & \left(T_{+} f_{+}, g_{+}\right)_{\mathfrak{H}_{+}}-\left(\Gamma_{1}^{+} f_{+}, \Gamma_{0}^{+} g_{+}\right)_{\mathcal{H}}+\left(\Gamma_{0}^{+} f_{+}, \Gamma_{1}^{+} g_{+}\right)_{\mathcal{H}} \\
& +\left(T_{-} f_{-}, g_{-}\right)_{\mathfrak{H}_{-}}-\left(\Gamma_{1}^{-} f_{-}, \Gamma_{0}^{-} g_{-}\right){ }_{\mathcal{H}}+\left(\Gamma_{0}^{-} f_{-}, \Gamma_{1}^{-} g_{-}\right) \mathcal{H}
\end{aligned}
$$


As $g \in \operatorname{dom}(A)$ one concludes that

$$
\Upsilon_{0} g=\Gamma_{0}^{+} g_{+}=\Gamma_{0}^{-} g_{-}, \quad \Upsilon_{1} g=\Gamma_{1}^{+} g_{+}=-\Gamma_{1}^{-} g_{-},
$$

by (4.7), and hence (4.17) takes on the form

$$
\begin{aligned}
\mathfrak{H}_{-2}\langle\mathcal{A} f, g\rangle_{\mathfrak{H}_{2}} & =(T f, g)_{\mathfrak{H}}-\left(\Gamma_{1}^{+} f_{+}+\Gamma_{1}^{-} f_{-}, \Upsilon_{0} g\right)_{\mathcal{H}}-\left(\Gamma_{0}^{-} f_{-}-\Gamma_{0}^{+} f_{+}, \Upsilon_{1} g\right)_{\mathcal{H}} \\
& =(T f, g)_{\mathfrak{H}}-\left(\left[\Gamma_{1} f\right], \Upsilon_{0} g\right)_{\mathcal{H}}-\left(\left[\Gamma_{0} f\right], \Upsilon_{1} g\right)_{\mathcal{H}} \\
& =\mathfrak{H}_{-2}\langle T f, g\rangle_{\mathfrak{H}_{2}}-\mathfrak{H}_{-2}\left\langle\Upsilon_{0}^{*}\left[\Gamma_{1} f\right], g\right\rangle_{\mathfrak{H}_{2}}-\mathfrak{H}_{-2}\left\langle\Upsilon_{1}^{*}\left[\Gamma_{0} f\right], g\right\rangle_{\mathfrak{H}_{2}},
\end{aligned}
$$

where (4.14)-(4.15) were used in the second equality, and (4.10) was employed in the last equality. Since (4.19) is true for all $g \in \operatorname{dom}(A)=\mathfrak{H}_{2}$ one concludes that

$$
\mathcal{A} f=T f-\Upsilon_{0}^{*}\left[\Gamma_{1} f\right]-\Upsilon_{1}^{*}\left[\Gamma_{0} f\right],
$$

and making use of the definition of $\mathcal{G}$ and (4.12)-(4.13) one finally obtains

$$
f=\mathcal{G} \mathcal{A} f=\mathcal{G} T f-\mathcal{G} \Upsilon_{0}^{*}\left[\Gamma_{1} f\right]-\mathcal{G} \Upsilon_{1}^{*}\left[\Gamma_{0} f\right]=\mathcal{G} T f-\mathcal{S}\left[\Gamma_{1} f\right]+\mathcal{D}\left[\Gamma_{0} f\right]
$$

as desired.

The following corollary may be viewed as an abstract unique continuation result.

Corollary 4.6 Under the assumptions of Theorem 4.5, the following assertions (i), (ii) hold:

(i) If $T_{+} f_{+}=0$ for some $f_{+} \in \operatorname{dom}\left(T_{+}\right)$satisfying $\Gamma_{0}^{+} f_{+}=\Gamma_{1}^{+} f_{+}=0$ then $f_{+}=0$.

(ii) If $T_{-} f_{-}=0$ for some $f_{-} \in \operatorname{dom}\left(T_{-}\right)$satisfying $\Gamma_{0}^{-} f_{-}=\Gamma_{1}^{-} f_{-}=0$ then $f_{-}=0$.

Proof We prove item (i), the proof of assertion (ii) being analogous. Assume that $T_{+} f_{+}=0$ for some $f_{+} \in \operatorname{dom}\left(T_{+}\right)$satisfying $\Gamma_{0}^{+} f_{+}=\Gamma_{1}^{+} f_{+}=0$. Setting $f_{-}:=0$ one obtains for $f:=\left(f_{+}, f_{-}\right)^{\top} \in \operatorname{dom}(T)$,

$$
T f=0, \quad\left[\Gamma_{0} f\right]=\Gamma_{0}^{-} f_{-}-\Gamma_{0}^{+} f_{+}=0, \quad\left[\Gamma_{1} f\right]=\Gamma_{1}^{+} f_{+}+\Gamma_{1}^{-} f_{-}=0 .
$$

Hence the third Green identity (4.16) implies $f=0$, therefore $f_{+}=0$.

\section{Coupling of Schrödinger operators on Lipschitz domains on manifolds}

In this section we illustrate the abstract material in Sects. 2-4 with the concrete case of Schrödinger operators on Lipschitz domains on boundaryless Riemannian manifolds, freely borrowing results from [4]. For more details and background information 
concerning differential geometry and partial differential equations on manifolds the interested reader is referred to [30,31,37], and the literature cited there.

Suppose $(M, g)$ is a compact, connected, $C^{\infty}$, boundaryless Riemannian manifold of (real) dimension $n \in \mathbb{N}$. In local coordinates, the metric tensor $g$ is expressed by

$$
g=\sum_{j, k=1}^{n} g_{j k} d x_{j} \otimes d x_{k}
$$

As is customary, we shall use the symbol $g$ to also abbreviate

$$
g:=\operatorname{det}\left[\left(g_{j k}\right)_{1 \leq j, k \leq n}\right]
$$

and we shall use $\left(g^{j k}\right)_{1 \leq j, k \leq n}$ to denote the inverse of the matrix $\left(g_{j k}\right)_{1 \leq j, k \leq n}$, that is,

$$
\left(g^{j k}\right)_{1 \leq j, k \leq n}:=\left[\left(g_{j k}\right)_{1 \leq j, k \leq n}\right]^{-1} .
$$

The volume element $d \mathcal{V}_{g}$ on $M$ with respect to the Riemannian metric $g$ in (5.1) then can be written in local coordinates as

$$
d \mathcal{V}_{g}(x)=\sqrt{g(x)} d^{n} x
$$

Following a common practice, we use $\left\{\partial_{j}\right\}_{1 \leq j \leq n}$ to denote a local basis in the tangent bundle $T M$ of the manifold $M$. This implies that if $X, Y \in T M$ are locally expressed as $X=\sum_{j=1}^{n} X_{j} \partial_{j}$ and $Y=\sum_{j=1}^{n} Y_{j} \partial_{j}$, then

$$
\langle X, Y\rangle_{T M}=\sum_{j, k=1}^{n} X_{j} Y_{k} g_{j k}
$$

where $\langle\cdot, \cdot\rangle_{T M}$ stands for the pointwise inner product in $T M$.

Next, we discuss the gradient and divergence operators associated with the metric $g$ on the manifold $M$. Specifically, given an open set $\Omega \subset M$ and some function $f \in C^{1}(\Omega)$, the gradient of $f$ is the vector field locally defined as

$$
\operatorname{grad}_{g}(f):=\sum_{j, k=1}^{n}\left(\partial_{j} f\right) g^{j k} \partial_{k}
$$

Also, given any vector field $X \in C^{1}(\Omega, T M)$ locally written as $X=\sum_{j=1}^{n} X_{j} \partial_{j}$, its divergence is given by

$$
\operatorname{div}_{g}(X):=\sum_{j=1}^{n} g^{-1 / 2} \partial_{j}\left(g^{1 / 2} X_{j}\right)=\sum_{j=1}^{n} \partial_{j} X_{j}+\sum_{j, k=1}^{n} \Gamma_{j k}^{j} X_{k},
$$


where $\Gamma_{j k}^{i}$ are the Christoffel symbols associated with the metric (5.1). The LaplaceBeltrami operator

$$
\Delta_{g}:=\operatorname{div}_{g} \operatorname{grad}_{g}
$$

is expressed locally as

$$
\Delta_{g} u=\sum_{j, k=1}^{n} g^{-1 / 2} \partial_{j}\left(g^{j k} g^{1 / 2} \partial_{k} u\right) .
$$

We are interested in working with the Schrödinger operator

$$
L:=-\Delta_{g}+V
$$

where the potential $V \in L^{\infty}(M)$ is a real scalar-valued function.

The reader is reminded that the scale of $L^{2}$-based Sobolev spaces $H^{S}(M)$ of fractional smoothness $s \in \mathbb{R}$ on $M$ may be defined in a natural fashion, via localization (using a smooth partition of unity subordinate to a finite cover of $M$ with local coordinate charts) and pull-pack to the Euclidean model. This scale of spaces is then adapted to an open subset $\Omega$ of $M$ via restriction, by setting

$$
H^{s}(\Omega):=\left\{\left.u\right|_{\Omega} \mid u \in H^{s}(M)\right\}, \quad s \in \mathbb{R}
$$

In particular, $H^{0}(\Omega)$ coincides with $L^{2}(\Omega)$, the space of square-integrable functions with respect to the volume element $d \mathcal{V}_{g}$ in $\Omega$. For each $s \in \mathbb{R}$ we also define

$$
\stackrel{\circ}{H}^{s}(\Omega):={\overline{C_{0}^{\infty}(\Omega)}}^{H^{s}(\Omega)}
$$

and equip the latter space with the norm inherited from $H^{s}(\Omega)$.

Since bounded Lipschitz domains in the Euclidean setting are invariant under $C^{1}$ diffeomorphisms (cf. [22]), this class may canonically be defined on the manifold $M$, using local coordinate charts. Given a Lipschitz domain $\Omega$, it is then possible to define (again, in a canonical manner, via localization and pull-back) fractional Sobolev spaces on its boundary, $H^{s}(\partial \Omega)$, for $s \in[-1,1]$. In such a scenario one has

$$
\left(H^{s}(\partial \Omega)\right)^{*}=H^{-s}(\partial \Omega), \quad s \in[-1,1]
$$

and $H^{0}(\partial \Omega)$ coincides with $L^{2}(\partial \Omega)$, the space of square-integrable functions with respect to the surface measure $\sigma_{g}$ induced by the ambient Riemannian metric on $\partial \Omega$. Moreover,

$$
\left\{\left.\phi\right|_{\partial \Omega} \mid \phi \in C^{\infty}(M)\right\} \text { is dense in each } H^{s}(\partial \Omega), \quad s \in[-1,1],
$$

and

$$
H^{s_{0}}(\partial \Omega) \hookrightarrow H^{s_{1}}(\partial \Omega) \text { continuously, whenever }-1 \leq s_{1} \leq s_{0} \leq 1 \text {. }
$$


In the following the operator $A$ in Sects. 3 and 4 will be the Schrödinger operator

$$
A:=-\Delta_{g}+V, \quad \operatorname{dom}(A):=H^{2}(M) .
$$

To proceed, we fix a Lipschitz domain $\Omega_{+} \subset M$ and denote

$$
\Omega_{-}:=M \backslash \overline{\Omega_{+}} \text {. }
$$

Then $\Omega_{-}$is also a Lipschitz domain, sharing a common compact boundary with $\Omega_{+}$,

$$
\mathcal{C}:=\partial \Omega_{+}=\partial \Omega_{-}
$$

At the global level, it is important to note that $A$ is selfadjoint in the Hilbert space $\mathfrak{H}=L^{2}(M)$. The decomposition of $M \backslash \mathcal{C}$ into the disjoint union of $\Omega_{+}$and $\Omega_{-}$, induces a direct orthogonal sum decomposition of $\mathfrak{H}$ into two Hilbert spaces $\mathfrak{H}_{+}$and $\mathfrak{H}_{-}$, defined as $\mathfrak{H}_{ \pm}:=L^{2}\left(\Omega_{ \pm}\right)$,

$$
\mathfrak{H}=L^{2}\left(\Omega_{+}\right) \oplus L^{2}\left(\Omega_{-}\right)
$$

In the following functions on $M$ will be identified with the pair of restrictions onto $\Omega_{+}$and $\Omega_{-}$and a vector notation will be used. For example, for $f \in L^{2}(M)$ we shall also write $\left(f_{+}, f_{-}\right)^{\top}$, where $f_{ \pm} \in L^{2}\left(\Omega_{ \pm}\right)$. This notation is in accordance with the notation in Sects. 3 and 4. Also, in the sequel we agree to abbreviate

$$
V_{ \pm}:=\left.V\right|_{\Omega_{ \pm}} \in L^{\infty}\left(\Omega_{ \pm}\right)
$$

For $s \geq 0$ we define the Banach spaces

$$
H_{\Delta}^{s}\left(\Omega_{ \pm}\right):=\left\{f_{ \pm} \in H^{s}\left(\Omega_{ \pm}\right) \mid \Delta_{g} f_{ \pm} \in L^{2}\left(\Omega_{ \pm}\right)\right\}
$$

equipped with the norms $\|\cdot\|_{H_{\Delta}^{s}\left(\Omega_{ \pm}\right)}$defined as

$$
\left\|f_{ \pm}\right\|_{H_{\Delta}^{s}\left(\Omega_{ \pm}\right)}:=\left\|f_{ \pm}\right\|_{H^{s}\left(\Omega_{ \pm}\right)}+\left\|\Delta_{g} f_{ \pm}\right\|_{L^{2}\left(\Omega_{ \pm}\right)}, \quad f_{ \pm} \in H_{\Delta}^{s}\left(\Omega_{ \pm}\right)
$$

The minimal and maximal realizations of $-\Delta_{g}+V_{ \pm}$in $L^{2}\left(\Omega_{ \pm}\right)$are defined by

$$
S_{\min , \pm}:=-\Delta_{g}+V_{ \pm}, \quad \operatorname{dom}\left(S_{\min , \pm}\right)=\stackrel{\circ}{H}^{2}\left(\Omega_{ \pm}\right)
$$

and

$$
S_{\max , \pm}:=-\Delta_{g}+V_{ \pm}, \quad \operatorname{dom}\left(S_{\max , \pm}\right)=H_{\Delta}^{0}\left(\Omega_{ \pm}\right) .
$$

In the next lemma we collect some well-known properties of the operators $S_{\min , \pm}$ and $S_{\max , \pm}$. A proof of this lemma and some further properties of the minimal and maximal realization of $-\Delta_{g}+V_{ \pm}$can be found, for instance, in [4]. 
Lemma 5.1 The operators $S_{\min , \pm}$ and $S_{\max , \pm}$ are densely defined and closed in $L^{2}\left(\Omega_{ \pm}\right)$. The operator $S_{\min , \pm}$ is symmetric, semibounded from below, and has infinite deficiency indices. Furthermore, $S_{\min , \pm}$ and $S_{\max , \pm}$ are adjoints of each other, that is,

$$
\left(S_{\min , \pm}\right)^{*}=S_{\max , \pm} \text { and } S_{\min , \pm}=\left(S_{\max , \pm}\right)^{*}
$$

Let $\mathfrak{n}^{ \pm} \in L^{\infty}(\mathcal{C}, T M)$ be the outward unit normal vectors to $\Omega_{ \pm}$. One observes that in the present situation $\mathfrak{n}^{+}=-\mathfrak{n}^{-}$. The Dirichlet and Neumann trace operators $\tau_{D}^{ \pm}$and $\tau_{N}^{ \pm}$, originally defined by

$$
\tau_{D}^{ \pm} f_{ \pm}:=f_{ \pm} \Upsilon_{\mathcal{C}}, \quad \tau_{N}^{ \pm} f_{ \pm}:=\left\langle\mathfrak{n}^{ \pm}, \operatorname{grad}_{g} f_{ \pm}\lceil\mathcal{C}\rangle_{T M}\right.
$$

for $f_{ \pm} \in C^{\infty}\left(\overline{\Omega_{ \pm}}\right)$, admit continuous linear extensions to operators

$$
\tau_{D}^{ \pm}: H_{\Delta}^{s}\left(\Omega_{ \pm}\right) \rightarrow H^{s-1 / 2}(\mathcal{C}) \text { and } \tau_{N}^{ \pm}: H_{\Delta}^{s}\left(\Omega_{ \pm}\right) \rightarrow H^{s-3 / 2}(\mathcal{C}),
$$

whose actions are compatible with one another, for all $s \in\left[\frac{1}{2}, \frac{3}{2}\right]$. We refer to [4] where it is also shown that

$$
\begin{aligned}
& \text { the trace operators } \tau_{D}^{ \pm}, \tau_{N}^{ \pm} \text {in }(5.27) \\
& \text { are both surjective for each } s \in\left[\frac{1}{2}, \frac{3}{2}\right] .
\end{aligned}
$$

We wish to augment (5.28) with the following density result.

Lemma 5.2 The ranges of the mappings

$$
\left\{f_{ \pm} \in H^{3 / 2}\left(\Omega_{ \pm}\right) \mid \Delta_{g} f_{ \pm} \in C^{\infty}\left(\overline{\Omega_{ \pm}}\right)\right\} \ni f_{ \pm} \mapsto\left(\tau_{D}^{ \pm} f_{ \pm},-\tau_{N}^{ \pm} f_{ \pm}\right)
$$

are dense in $L^{2}(\mathcal{C}) \times L^{2}(\mathcal{C})$.

The proof of Lemma 5.2 requires some preparations. To get started, we fix two potentials $0 \leq V_{0}^{ \pm} \in C^{\infty}(M)$ which are not identically zero on $M$, and which vanish on $\Omega_{+}$, and on $\Omega_{-}$, respectively. Then (cf. [35, p. 27]) for each $s \in[0,2]$, the operators

$$
-\Delta_{g}+V_{0}^{ \pm}: H^{2-s}(M) \longrightarrow H^{-s}(M)
$$

are invertible, with bounded inverses.

Furthermore, for each choice of sign, the said inverses act in a compatible fashion with one another. Abbreviating $\mathcal{G}_{0}^{ \pm}:=\left(-\Delta_{g}+V_{0}^{ \pm}\right)^{-1}$ then yields two well-defined, linear, and bounded operators

$$
\mathcal{G}_{0}^{ \pm}: H^{-s}(M) \longrightarrow H^{2-s}(M) \text { for each } s \in[0,2] .
$$

The Schwartz kernels of these operators are distributions $E_{0}^{ \pm}$on $M \times M$ which are smooth outside of the $\operatorname{diagonal} \operatorname{diag} M:=\{(x, x): x \in M\}$. In particular, it makes 
sense to talk about pointwise values $E_{0}^{ \pm}(x, y)$ for $x, y \in M$ with $x \neq y$. Among other things, the functions $E_{0}^{ \pm}(\cdot, \cdot) \in C^{\infty}((M \times M) \backslash \operatorname{diag} M)$ satisfy

$$
E_{0}^{ \pm}(x, y)=E_{0}^{ \pm}(y, x) \text { for all } x, y \in M \text { with } x \neq y
$$

At this stage, we bring in the single and double layer potentials on Lipschitz domains on manifolds considered in [33-35]. Their actions on an arbitrary function $\varphi \in L^{2}(\mathcal{C})$ are, respectively,

$$
\left(\mathcal{S}_{0}^{ \pm} \varphi\right)(x):=\int_{\mathcal{C}} E_{0}^{ \pm}(x, y) \varphi(y) d \sigma_{g}(y), \quad x \in \Omega_{ \pm}
$$

and

$$
\left(\mathcal{D}_{0}^{ \pm} \varphi\right)(x):= \pm \int_{\mathcal{C}}\left\langle\mathfrak{n}^{ \pm}(y), \operatorname{grad}_{g_{y}}\left[E_{0}^{ \pm}(x, y)\right]\right\rangle_{T_{y} M} \varphi(y) d \sigma_{g}(y), \quad x \in \Omega_{ \pm},
$$

where $\sigma_{g}$ is the surface measure induced by the ambient Riemannian metric on $\mathcal{C}$. Let us also consider their boundary versions, that is, the singular integral operators acting on an arbitrary function $\varphi \in L^{2}(\mathcal{C})$ according to

$$
\left(S_{0}^{ \pm} \varphi\right)(x):=\int_{\mathcal{C}} E_{0}^{ \pm}(x, y) \varphi(y) d \sigma_{g}(y), \quad x \in \mathcal{C},
$$

and

$$
\left(K_{0}^{ \pm} \varphi\right)(x):=\mathrm{P} . \mathrm{V} \cdot \int_{\mathcal{C}}\left\langle\mathfrak{n}^{ \pm}(y), \operatorname{grad}_{g_{y}}\left[E_{0}^{ \pm}(x, y)\right]\right\rangle_{T_{y} M} \varphi(y) d \sigma_{g}(y), \quad x \in \mathcal{C},
$$

where P.V. indicates that the integral is considered in the principal value sense (i.e., removing a small geodesic ball centered at the singularity and passing to the limit as its radius shrinks to zero). Work in [33-35] ensures that the following properties hold:

$$
\begin{aligned}
& \mathcal{D}_{0}^{ \pm}: L^{2}(\mathcal{C}) \rightarrow H_{\Delta}^{1 / 2}\left(\Omega_{ \pm}\right) \text {are linear and bounded operators, } \\
& \left(-\Delta_{g}+V_{0}^{ \pm}\right)\left(\mathcal{D}_{0}^{ \pm} \varphi\right)=0 \text { in } \Omega_{ \pm}, \text {for every function } \varphi \in L^{2}(\mathcal{C}), \\
& K_{0}^{ \pm}: L^{2}(\mathcal{C}) \rightarrow L^{2}(\mathcal{C}) \text { are linear and bounded operators, } \\
& \tau_{D}^{ \pm}\left(\mathcal{D}_{0}^{ \pm} \varphi\right)= \pm\left(\frac{1}{2} I+K_{0}^{ \pm}\right) \varphi \text { on } \mathcal{C} \text {, for each } \varphi \in L^{2}(\mathcal{C}), \\
& \mathcal{S}_{0}^{ \pm}: L^{2}(\mathcal{C}) \rightarrow H_{\Delta}^{3 / 2}\left(\Omega_{ \pm}\right) \text {are linear and bounded operators, } \\
& \left(-\Delta_{g}+V_{0}^{ \pm}\right)\left(\mathcal{S}_{0}^{ \pm} \varphi\right)=0 \text { in } \Omega_{ \pm}, \text {for every function } \varphi \in L^{2}(\mathcal{C}), \\
& S_{0}^{ \pm}: L^{2}(\mathcal{C}) \rightarrow L^{2}(\mathcal{C}) \text { are linear, bounded, self-adjoint, and injective, } \\
& \tau_{D}^{ \pm}\left(\mathcal{S}_{0}^{ \pm} \varphi\right)=S_{0}^{ \pm} \varphi \text { on } \mathcal{C}, \text { for each function } \varphi \in L^{2}(\mathcal{C}), \\
& \tau_{N}^{ \pm}\left(\mathcal{S}_{0}^{ \pm} \varphi\right)=\left(-\frac{1}{2} I+\left(K_{0}^{ \pm}\right)^{*}\right) \varphi \text { on } \mathcal{C} \text {, for every } \varphi \in L^{2}(\mathcal{C}),
\end{aligned}
$$


where $I$ is the identity operator on $L^{2}(\mathcal{C})$, and $\left(K_{0}^{ \pm}\right)^{*}$ are the adjoints of the operators $K_{0}^{ \pm}$in (5.40). After this preamble, we are ready to present the proof of Lemma 5.2.

Proof of Lemma 5.2 The density results claimed in the statement follow as soon as we establish that whenever two functions $h_{D}, h_{N} \in L^{2}(\mathcal{C})$ satisfy

$$
\begin{aligned}
& \left(\tau_{D}^{ \pm} f_{ \pm}, h_{D}\right)_{L^{2}(\mathcal{C})}-\left(\tau_{N}^{ \pm} f_{ \pm}, h_{N}\right)_{L^{2}(\mathcal{C})}=0 \\
& \quad \text { for all } f_{ \pm} \in H^{3 / 2}\left(\Omega_{ \pm}\right) \text {with } \Delta_{g} f_{ \pm} \in C^{\infty}\left(\overline{\Omega_{ \pm}}\right),
\end{aligned}
$$

then necessarily $h_{D}=0$ and $h_{N}=0$. To this end, pick an arbitrary $h \in L^{2}(\mathcal{C})$ and consider $f_{ \pm}:=\mathcal{S}_{0}^{ \pm} h$ in $\Omega_{ \pm}$. Then $f_{ \pm} \in H_{\Delta}^{3 / 2}\left(\Omega_{ \pm}\right)$due to (5.42). Also, relying on (5.43) and the fact that, by design, the potentials $V_{0}^{ \pm}$vanish in $\Omega_{ \pm}$, we may write $\Delta_{g} f_{ \pm}=\left(-\Delta_{g}+V_{0}^{ \pm}\right) f_{ \pm}=0$ in $\Omega_{ \pm}$. Granted these properties of $f_{ \pm}$, from (5.47), (5.45), (5.46), and (5.44), one concludes that

$$
\begin{aligned}
0 & =\left(\tau_{D}^{ \pm} f_{ \pm}, h_{D}\right)_{L^{2}(\mathcal{C})}-\left(\tau_{N}^{ \pm} f_{ \pm}, h_{N}\right)_{L^{2}(\mathcal{C})} \\
& =\left(S_{0}^{ \pm} h, h_{D}\right)_{L^{2}(\mathcal{C})}-\left(\left(-\frac{1}{2} I+\left(K_{0}^{ \pm}\right)^{*}\right) h, h_{N}\right)_{L^{2}(\mathcal{C})} \\
& =\left(h, S_{0}^{ \pm} h_{D}\right)_{L^{2}(\mathcal{C})}-\left(h,\left(-\frac{1}{2} I+K_{0}^{ \pm}\right) h_{N}\right)_{L^{2}(\mathcal{C})} \\
& =\left(h, S_{0}^{ \pm} h_{D}-\left(-\frac{1}{2} I+K_{0}^{ \pm}\right) h_{N}\right)_{L^{2}(\mathcal{C})} .
\end{aligned}
$$

With this in hand, the arbitrariness of $h \in L^{2}(\mathcal{C})$ then forces

$$
S_{0}^{ \pm} h_{D}-\left(-\frac{1}{2} I+K_{0}^{ \pm}\right) h_{N}=0 .
$$

Next, we pick two arbitrary functions $\phi_{ \pm} \in C_{0}^{\infty}\left(\Omega_{ \pm}\right)$and, this time, consider $f_{ \pm}:=\mathcal{G}_{0}^{ \pm} \phi_{ \pm}$in $\Omega_{ \pm}$. Then (5.32) ensures that $f_{ \pm} \in H^{2}\left(\Omega_{ \pm}\right)$. Given that, by design, $V_{0}^{ \pm}$vanish in $\Omega_{ \pm}$, one also has $\Delta_{g} f_{ \pm}=\left(-\Delta_{g}+V_{0}^{ \pm}\right) \mathcal{G}_{0}^{ \pm} \phi_{ \pm}=\phi_{ \pm}$in $\Omega_{ \pm}$. Having established these properties of $f_{ \pm},(5.47)$ implies that

$$
\left(\tau_{D}^{ \pm} f_{ \pm}, h_{D}\right)_{L^{2}(\mathcal{C})}-\left(\tau_{N}^{ \pm} f_{ \pm}, h_{N}\right)_{L^{2}(\mathcal{C})}=0
$$

Now we take a closer look at the two terms in the left-hand side of (5.51). For the first term we write

$$
\begin{aligned}
\left(\tau_{D}^{ \pm} f_{ \pm}, h_{D}\right)_{L^{2}(\mathcal{C})} & =\int_{\mathcal{C}}\left(\int_{\Omega_{ \pm}} E_{0}^{ \pm}(x, y) \overline{\phi_{ \pm}(y)} d \mathcal{V}_{g}(y)\right) h_{D}(x) d \sigma_{g}(x) \\
& =\int_{\Omega_{ \pm}}\left(\int_{\mathcal{C}} E_{0}^{ \pm}(x, y) h_{D}(x) d \sigma_{g}(x)\right) \overline{\phi_{ \pm}(y)} d \mathcal{V}_{g}(y) \\
& =\int_{\Omega_{ \pm}}\left(\mathcal{S}_{0}^{ \pm} h_{D}\right)(y) \overline{\phi_{ \pm}(y)} d \mathcal{V}_{g}(y)
\end{aligned}
$$

where the first equality uses the definition of $E_{0}^{ \pm}(x, y)$, the second equality is based on Fubini's theorem, while the third equality is a consequence of (5.33) and (5.34). For the second term in (5.51) we compute 


$$
\begin{aligned}
& \left(\tau_{N}^{ \pm} f_{ \pm}, h_{N}\right)_{L^{2}(\mathcal{C})} \\
& \quad=\int_{\mathcal{C}}\left(\int_{\Omega_{ \pm}}\left\langle\mathfrak{n}^{ \pm}(x), \operatorname{grad}_{g_{x}}\left[E_{0}^{ \pm}(x, y)\right]\right\rangle_{T_{x} M} \overline{\phi_{ \pm}(y)} d \mathcal{V}_{g}(y)\right) h_{N}(x) d \sigma_{g}(x) \\
& \quad=\int_{\Omega_{ \pm}}\left(\int_{\mathcal{C}}\left\langle\mathfrak{n}^{ \pm}(x), \operatorname{grad}_{g_{x}}\left[E_{0}^{ \pm}(x, y)\right]\right\rangle_{T_{x} M} h_{N}(x) d \sigma_{g}(x)\right) \overline{\phi_{ \pm}(y)} d \mathcal{V}_{g}(y) \\
& \quad= \pm \int_{\Omega_{ \pm}}\left(\mathcal{D}_{0}^{ \pm} h_{N}\right)(y) \overline{\phi_{ \pm}(y)} d \mathcal{V}_{g}(y),
\end{aligned}
$$

where the first equality relies on the definition of $E_{0}^{ \pm}(x, y)$, the second equality uses Fubini's theorem, while the third equality is implied by (5.33) and (5.35).

Together, (5.51), (5.52), and (5.53) imply that

$$
\int_{\Omega_{ \pm}}\left\{\left(\mathcal{S}_{0}^{ \pm} h_{D}\right)(y) \mp\left(\mathcal{D}_{0}^{ \pm} h_{N}\right)(y)\right\} \overline{\phi_{ \pm}(y)} d \mathcal{V}_{g}(y)=0
$$

which, in view of the arbitrariness of $\phi_{ \pm} \in C_{0}^{\infty}\left(\Omega_{ \pm}\right)$, forces

$$
\mathcal{S}_{0}^{ \pm} h_{D} \mp \mathcal{D}_{0}^{ \pm} h_{N}=0 \text { in } \Omega_{ \pm}
$$

Applying $\tau_{D}^{ \pm}$to both sides of (5.55) then yields, on account of (5.45) and (5.41),

$$
S_{0}^{ \pm} h_{D}-\left(\frac{1}{2} I+K_{0}^{ \pm}\right) h_{N}=0 \text { on } \mathcal{C}
$$

The end-game in the proof of the lemma is as follows. Subtracting (5.56) from (5.50) proves that $h_{N}=0$. Using this back into (5.56) leads to $S_{0}^{ \pm} h_{D}=0$ which, in light of the injectivity of the single layer operators in (5.44), shows that $h_{D}=0$ as well. Hence, $h_{D}=h_{N}=0$, as desired.

Going further, in the next theorem we define quasi boundary triples for $S_{\max , \pm}=$ $\left(S_{\min , \pm}\right)^{*}$ with the natural trace maps as boundary maps defined on the domain of the operators

$$
T_{ \pm}:=-\Delta_{g}+V_{ \pm}, \quad \operatorname{dom}\left(T_{ \pm}\right):=H_{\Delta}^{3 / 2}\left(\Omega_{ \pm}\right),
$$

in $L^{2}\left(\Omega_{ \pm}\right)$. One recalls that

$$
T=\left(\begin{array}{cc}
T_{+} & 0 \\
0 & T_{-}
\end{array}\right) \text {in } L^{2}(M)=L^{2}\left(\Omega_{+}\right) \oplus L^{2}\left(\Omega_{-}\right) .
$$

With this choice of boundary maps the values of the corresponding Weyl function are Dirichlet-to-Neumann maps (up to a minus sign).

Theorem 5.3 Let $\Omega_{ \pm}$and $T_{ \pm}$be as above, and let

$$
\Gamma_{0}^{ \pm}, \Gamma_{1}^{ \pm}: H_{\Delta}^{3 / 2}\left(\Omega_{ \pm}\right) \rightarrow L^{2}(\mathcal{C}), \quad \Gamma_{0}^{ \pm} f_{ \pm}:=\tau_{D}^{ \pm} f_{ \pm}, \quad \Gamma_{1}^{ \pm} f_{ \pm}:=-\tau_{N}^{ \pm} f_{ \pm}
$$


Then $\left\{L^{2}(\mathcal{C}), \Gamma_{0}^{ \pm}, \Gamma_{1}^{ \pm}\right\}$are quasi boundary triples for $T_{ \pm} \subset S_{\max , \pm}$ such that

$$
S_{\min , \pm}=T_{ \pm} \uparrow\left(\operatorname{ker}\left(\Gamma_{0}^{ \pm}\right) \cap \operatorname{ker}\left(\Gamma_{1}^{ \pm}\right)\right)
$$

In addition, the following statements (i)-(iii) hold:

(i) The Dirichlet realizations $A_{D, \pm}$ and the Neumann realizations $A_{N, \pm}$ of $-\Delta_{g}+$ $V_{ \pm}$in $L^{2}\left(\Omega_{ \pm}\right)$coincide with $A_{0, \pm}=T \uparrow \operatorname{ker}\left(\Gamma_{0}^{ \pm}\right)$and $A_{1, \pm}=T \uparrow \operatorname{ker}\left(\Gamma_{1}^{ \pm}\right)$, respectively,

$$
\begin{aligned}
& A_{D, \pm}=T_{ \pm} \uparrow \operatorname{ker}\left(\Gamma_{0}^{ \pm}\right)=\left(-\Delta_{g}+V_{ \pm}\right) \uparrow\left\{f_{ \pm} \in H_{\Delta}^{3 / 2}\left(\Omega_{ \pm}\right) \mid \tau_{D}^{ \pm} f_{ \pm}=0\right\} \\
& A_{N, \pm}=T_{ \pm}\left\lceil\operatorname{ker}\left(\Gamma_{1}^{ \pm}\right)=\left(-\Delta_{g}+V_{ \pm}\right) \uparrow\left\{f_{ \pm} \in H_{\Delta}^{3 / 2}\left(\Omega_{ \pm}\right) \mid \tau_{N}^{ \pm} f_{ \pm}=0\right\}\right.
\end{aligned}
$$

and both operators are self-adjoint in $L^{2}\left(\Omega_{ \pm}\right)$.

(ii) The values $\gamma_{ \pm}(z): L^{2}(\mathcal{C}) \supset H^{1}(\mathcal{C}) \rightarrow L^{2}\left(\Omega_{ \pm}\right)$of the $\gamma$-fields are given by

$$
\gamma_{ \pm}(z) \varphi=f_{ \pm}, \quad \varphi \in H^{1}(\mathcal{C}), \quad z \in \rho\left(A_{D, \pm}\right)
$$

where $f_{ \pm} \in L^{2}\left(\Omega_{ \pm}\right)$are the unique solutions of the boundary value problems

$$
f_{ \pm} \in H_{\Delta}^{3 / 2}\left(\Omega_{ \pm}\right), \quad\left(-\Delta_{g}+V_{ \pm}-z\right) f_{ \pm}=0, \quad \tau_{D}^{ \pm} f_{ \pm}=\varphi
$$

(iii) The values $M_{ \pm}(z): L^{2}(\mathcal{C}) \supset H^{1}(\mathcal{C}) \rightarrow L^{2}(\mathcal{C})$ of the Weyl functions are Dirichlet-to-Neumann maps, given by

$$
M_{ \pm}(z) \varphi=-\tau_{N}^{ \pm} f_{ \pm}, \quad \varphi \in H^{1}(\mathcal{C}), \quad z \in \rho\left(A_{D, \pm}\right)
$$

where $f_{ \pm}=\gamma_{ \pm}(z) \varphi$ are the unique solutions of (5.64).

Proof Let us verify the properties stipulated in Definition 2.1 in the current case. First, the abstract Green identity (2.1) presently corresponds to the second Green identity for the Schrödinger operator (5.10) on the Lipschitz domain $\Omega$, proved in [4]. Second, the fact that $\operatorname{ran}\left(\Gamma_{0}^{ \pm}, \Gamma_{1}^{ \pm}\right)^{\top}$ is dense in $L^{2}(\mathcal{C}) \times L^{2}(\mathcal{C})$ is readily implied by Lemma 5.2 [bearing in mind (5.21)]. Third, the self-adjointness of $A_{0, \pm}=T_{ \pm} \uparrow \operatorname{ker}\left(\Gamma_{0}^{ \pm}\right)$is clear from the fact that these operators coincide with the self-adjoint Dirichlet realizations of $-\Delta_{g}+V_{ \pm}$in $\Omega_{ \pm}$studied in [4].

Fourth, we focus on establishing that $\bar{T}_{ \pm}=S_{\max , \pm}$. In turn, since dom $\left(T_{ \pm}\right)$contains $\operatorname{dom}\left(A_{D, \pm}\right)+\operatorname{dom}\left(A_{N, \pm}\right)$, this is going to be a consequence of the fact that

$$
\begin{aligned}
& \operatorname{dom}\left(A_{D, \pm}\right)+\operatorname{dom}\left(A_{N, \pm}\right) \text { is dense in } \operatorname{dom}\left(S_{\text {max }, \pm}\right) \\
& \text { with respect to the graph norm. }
\end{aligned}
$$


To prove (5.66), we assume that $h \in \operatorname{dom}\left(S_{\max , \pm}\right)$ is such that

$$
\begin{aligned}
& \left(f_{D}+f_{N}, h\right)_{L^{2}\left(\Omega_{ \pm}\right)}+\left(S_{\max , \pm}\left(f_{D}+f_{N}\right), S_{\max , \pm} h\right)_{L^{2}\left(\Omega_{ \pm}\right)}=0 \\
& \quad \text { for all } f_{D} \in \operatorname{dom}\left(A_{D, \pm}\right), f_{N} \in \operatorname{dom}\left(A_{N, \pm}\right)
\end{aligned}
$$

Then

$$
\left(A_{D, \pm} f_{D}, S_{\max , \pm} h\right)_{L^{2}\left(\Omega_{ \pm}\right)}=\left(f_{D},-h\right)_{L^{2}\left(\Omega_{ \pm}\right)}, \quad f_{D} \in \operatorname{dom}\left(A_{D, \pm}\right)
$$

and

$$
\left(A_{N, \pm} f_{N}, S_{\max , \pm} h\right)_{L^{2}\left(\Omega_{ \pm}\right)}=\left(f_{N},-h\right)_{L^{2}\left(\Omega_{ \pm}\right)}, \quad f_{N} \in \operatorname{dom}\left(A_{N, \pm}\right) .
$$

Together, (5.68) and (5.69) prove that

$$
\begin{aligned}
& S_{\text {max }, \pm} h \in \operatorname{dom}\left(A_{D, \pm}\right) \cap \operatorname{dom}\left(A_{N, \pm}\right)=\operatorname{dom}\left(S_{\min , \pm}\right) \\
& \quad \text { and } S_{\min , \pm} S_{\max , \pm} h=-h .
\end{aligned}
$$

Finally, from

$$
\begin{aligned}
0 & =\left(\left(I+S_{\min , \pm} S_{\max , \pm}\right) h, h\right)_{L^{2}\left(\Omega_{ \pm}\right)} \\
& =(h, h)_{L^{2}\left(\Omega_{ \pm}\right)}+\left(S_{\max , \pm} h, S_{\max , \pm} h\right)_{L^{2}\left(\Omega_{ \pm}\right)},
\end{aligned}
$$

one concludes that $h=0$. Hence (5.66) holds, completing the proof of the fact that $\bar{T}_{ \pm}=S_{\max , \pm}$.

This shows that $\left\{L^{2}(\mathcal{C}), \Gamma_{0}^{ \pm}, \Gamma_{1}^{ \pm}\right\}$are indeed quasi boundary triples for $T_{ \pm}$. That $T_{ \pm} \subset S_{\text {max }, \pm}$ is clear from definitions, while (5.60) has been established in [4].

Thanks to work in [4], the assertions in (ii) and (iii) follow immediately from the definition of the $\gamma$-field and the Weyl function. We refer the interested reader to [4] for more details. Here we only wish to note that in the case of a bounded Lipschitz domain in the flat Euclidean setting (i.e., $\mathbb{R}^{n}$ equipped with the standard metric) a similar result has been established in [8, Theorem 4.1].

In the following we establish the link to the coupling procedure discussed in Sect. 3. First of all we set $\mathcal{H}:=L^{2}(\mathcal{C})$ so that the quasi boundary triples in Sect. 3 are those in Theorem 5.3. The operator $S$ in (3.4) is the direct orthogonal sum of the minimal realizations $S_{\min ,+}$ and $S_{\min ,-}$,

$$
S=\left(\begin{array}{cc}
S_{\min ,+} & 0 \\
0 & S_{\min ,-}
\end{array}\right),
$$

and the boundary mappings in the quasi boundary triple $\left\{L^{2}(\mathcal{C}) \oplus L^{2}(\mathcal{C}), \Gamma_{0}, \Gamma_{1}\right\}$ in (3.7) are now given by

$$
\Gamma_{0} f=\left(\begin{array}{l}
\tau_{D}^{+} f_{+} \\
\tau_{D}^{-} f_{-}
\end{array}\right) \quad \text { and } \quad \Gamma_{1} f=\left(\begin{array}{l}
-\tau_{N}^{+} f_{+} \\
-\tau_{N}^{-} f_{-}
\end{array}\right),
$$


where $f=\left(f_{+}, f_{-}\right) \in \operatorname{dom}(T)$ with $T_{ \pm}, T$ given as in (5.57), (5.58). The self-adjoint operator corresponding to $\operatorname{ker}\left(\Gamma_{0}\right)$ is the orthogonal sum of the Dirichlet operators $A_{D,+}$ and $A_{D,-}$ in $L^{2}\left(\Omega_{+}\right)$and $L^{2}\left(\Omega_{-}\right)$, respectively,

$$
A_{0}=\left(\begin{array}{cc}
A_{D,+} & 0 \\
0 & A_{D,-}
\end{array}\right) .
$$

The following lemma shows that the coupling of the quasi boundary triples $\left\{L^{2}(\mathcal{C}), \Gamma_{0}^{+}, \Gamma_{1}^{+}\right\}$and $\left\{L^{2}(\mathcal{C}), \Gamma_{0}^{-}, \Gamma_{1}^{-}\right\}$in Theorem 3.1 leads to the self-adjoint Schrödinger operator in (5.16).

Lemma 5.4 The operator

$$
T \uparrow\left\{f=\left(f_{+}, f_{-}\right)^{\top} \in \operatorname{dom}(T) \mid \Gamma_{0}^{+} f_{+}=\Gamma_{0}^{-} f_{-}, \Gamma_{1}^{+} f_{+}=-\Gamma_{1}^{-} f_{-}\right\}
$$

coincides with the self-adjoint operator A in (5.16).

Proof Since any function $f \in H^{2}(M)$ satisfies

$$
\begin{aligned}
& \Gamma_{0}^{+} f_{+}=\tau_{D}^{+} f_{+}=\tau_{D}^{-} f_{-}=\Gamma_{0}^{-} f_{-}, \\
& \Gamma_{1}^{+} f_{+}=-\tau_{N}^{+} f_{+}=\tau_{N}^{-} f_{-}=-\Gamma_{1}^{-} f_{-},
\end{aligned}
$$

and $f_{ \pm} \in H^{2}\left(\Omega_{ \pm}\right) \subset H_{\Delta}^{3 / 2}\left(\Omega_{ \pm}\right)=\operatorname{dom}\left(T_{ \pm}\right)$, it follows that $H^{2}(M)=\operatorname{dom}(A)$ is contained in the domain of the operator in (5.75). On the other hand, it follows from Theorem 3.1 (i) that the operator in (5.75) is symmetric, and hence self-adjoint (as it extends the self-adjoint operator $A$ ).

As an immediate consequence of the observation in Lemma 5.4 we obtain the next corollary. First, we note that the self-adjointness of the operator $A$ in (5.16), Theorem 3.1, and the fact that

$$
A=T \nmid\left\{f=\left(f_{+}, f_{-}\right)^{\top} \in \operatorname{dom}(T) \mid \Gamma_{0}^{+} f_{+}=\Gamma_{0}^{-} f_{-}, \Gamma_{1}^{+} f_{+}=-\Gamma_{1}^{-} f_{-}\right\},
$$

imply

$$
\operatorname{ran}\left(\Gamma_{1}^{ \pm} \uparrow \operatorname{dom}\left(A_{D, \pm}\right)\right) \subseteq \operatorname{ran}\left(M_{+}(z)+M_{-}(z)\right), \quad z \in \mathbb{C}_{+} \cup \mathbb{C}_{-}
$$

where $M_{ \pm}$are (minus) the Dirichlet-to-Neumann maps in (5.65).

Corollary 5.5 Let $A_{0}$ be the orthogonal sum of the Dirichlet operators in (5.74), let $M_{ \pm}$be the (minus) Dirichlet-to-Neumann maps in (5.65) and let $\gamma$ be the orthogonal sum of the $\gamma$-fields in (5.63) [cf. (3.9)]. For all $z \in \rho(A) \cap \rho\left(A_{0}\right)$, the resolvent of $A$ is given by

$$
\left(A-z I_{L^{2}(M)}\right)^{-1}=\left(A_{0}-z I_{L^{2}(M)}\right)^{-1}+\gamma(z) \Theta(z) \gamma(\bar{z})^{*},
$$


where

$$
\Theta(z):=\left(\begin{array}{ll}
-\left(M_{+}(z)+M_{-}(z)\right)^{-1} & -\left(M_{+}(z)+M_{-}(z)\right)^{-1} \\
-\left(M_{+}(z)+M_{-}(z)\right)^{-1} & -\left(M_{+}(z)+M_{-}(z)\right)^{-1}
\end{array}\right) .
$$

In a similar way one obtains a representation for the resolvent of $A$ from Theorem 3.3, where $\left(A-z I_{L^{2}(M)}\right)^{-1}$ is compared with the orthogonal sum of the self-adjoint Dirichlet operator $A_{D,+}$ in $L^{2}\left(\Omega_{+}\right)$and the self-adjoint Neumann operator $A_{N,-}$ in $L^{2}\left(\Omega_{-}\right)$.

Corollary 5.6 Let $M_{ \pm}$be the (minus) Dirichlet-to-Neumann maps in (5.65) and let $\gamma_{ \pm}$be the $\gamma$-fields in (5.63). For all $z \in \rho(A) \cap \rho\left(A_{D,+}\right) \cap \rho\left(A_{D,-}\right) \cap \rho\left(A_{N,-}\right)$, the resolvent of $A$ is given by

$$
\left(A-z I_{L^{2}(M)}\right)^{-1}=\left(\left(\begin{array}{cc}
A_{D,+} & 0 \\
0 & A_{N,-}
\end{array}\right)-z I_{L^{2}(M)}\right)^{-1}+\widehat{\gamma}(z) \Sigma(z) \widehat{\gamma}(\bar{z})^{*},
$$

where

$$
\widehat{\gamma}(z):=\left(\begin{array}{cc}
\gamma_{+}(z) & 0 \\
0 & \gamma_{-}(z) M_{-}(z)^{-1}
\end{array}\right), \quad \Sigma(z):=-\left(\begin{array}{cc}
M_{+}(z) & I_{L^{2}(\mathcal{C})} \\
I_{L^{2}(\mathcal{C})} & -M_{-}(z)^{-1}
\end{array}\right)^{-1} .
$$

Our next aim is to illustrate the abstract third Green identity from Sect. 4 in the present context of Schrödinger operators on Lipschitz domains on smooth, boundaryless Riemannian manifolds. Since $\operatorname{dom}(A)=H^{2}(M)$ and the graph norm (4.2) is equivalent to the usual norm on the Sobolev space $H^{2}(M)$ we have $\mathfrak{H}_{2}=H^{2}(M)$ and hence the Gelfand triple in (4.5) is of the form

$$
H^{2}(M) \hookrightarrow L^{2}(M) \hookrightarrow H^{-2}(M)
$$

Using the notation in (4.7) we note that the mappings

$$
\Upsilon_{0}=\tau_{D}^{+}: H^{2}(M) \rightarrow L^{2}(\mathcal{C}), \quad \Upsilon_{1}=-\tau_{N}^{+}: H^{2}(M) \rightarrow L^{2}(\mathcal{C})
$$

are bounded, which is clearly in accordance (and also follows from) Lemma 4.2. Thus, the dual operators

$$
\Upsilon_{0}^{*}=\left(\tau_{D}^{+}\right)^{*}: L^{2}(\mathcal{C}) \rightarrow H^{-2}(M), \quad \Upsilon_{1}^{*}=\left(-\tau_{N}^{+}\right)^{*}: L^{2}(\mathcal{C}) \rightarrow H^{-2}(M)
$$

are bounded. Moreover, under the additional assumption that

$$
V \geq 0 \text { and not identically zero on } M \text {, }
$$

it has been proved in $[35$, p. 27] that

$$
-\Delta_{g}+V: L^{2}(M) \rightarrow H^{-2}(M) \text { is invertible, with bounded inverse. }
$$


In such a scenario, if $\mathcal{G}: H^{-2}(M) \rightarrow L^{2}(M)$ denotes the inverse of (5.88), it follows that

$$
\mathcal{G}\left(-\Delta_{g}+V\right) f=f, \quad f \in L^{2}(M) .
$$

Then the (abstract) single and double layer potentials in (4.12) and (4.13) are given by

$$
\mathcal{S}: L^{2}(\mathcal{C}) \rightarrow L^{2}(M), \quad \varphi \mapsto \mathcal{G} \Upsilon_{0}^{*} \varphi=\mathcal{G}\left(\tau_{D}^{+}\right)^{*} \varphi,
$$

and

$$
\mathcal{D}: L^{2}(\mathcal{C}) \rightarrow L^{2}(M), \quad \varphi \mapsto-\mathcal{G} \Upsilon_{1}^{*} \varphi=\mathcal{G}\left(\tau_{N}^{+}\right)^{*} \varphi .
$$

Moreover, if $E(x, y)$ is the integral kernel of $\mathcal{G}$, then the action of these abstract single and double layer potentials on a function $\varphi \in L^{2}(\mathcal{C})$ may be explicitly written as

$$
(\mathcal{S} \varphi)(x)=\int_{\mathcal{C}} E(x, y) \varphi(y) d \sigma_{g}(y), \quad x \in M,
$$

and

$$
(\mathcal{D} \varphi)(x)=\int_{\mathcal{C}}\left\langle\mathfrak{n}^{+}(y), \operatorname{grad}_{g_{y}} E(x, y)\right\rangle_{T_{y} M} \varphi(y) d \sigma_{g}(y), \quad x \in M,
$$

which are in agreement with (5.34), (5.35). Furthermore, the abstract jump relations in (4.14)-(4.15) are

$$
\begin{aligned}
& {\left[\Gamma_{0} f\right]=\Gamma_{0}^{-} f_{-}-\Gamma_{0}^{+} f_{+}=\tau_{D}^{-} f_{-}-\tau_{D}^{+} f_{+}, \quad f=\left(f_{+}, f_{-}\right)^{\top} \in \operatorname{dom}(T),} \\
& {\left[\Gamma_{1} f\right]=\Gamma_{1}^{+} f_{+}+\Gamma_{1}^{-} f_{-}=-\tau_{N}^{+} f_{+}-\tau_{N}^{-} f_{-}, \quad f=\left(f_{+}, f_{-}\right)^{\top} \in \operatorname{dom}(T) .}
\end{aligned}
$$

As a consequence of the above considerations and Theorem 4.5 we obtain the following version of Green's third identity for the Schrödinger operator $-\Delta_{g}+V$ on $M$.

Theorem 5.7 With the fundamental solution operator $\mathcal{G}$ in (5.89), the layer potentials in (5.90)-(5.91), and the jump relations in (5.94)-(5.95), one has

$$
f=\mathcal{G} T f+\mathcal{D}\left[\Gamma_{0} f\right]-\mathcal{S}\left[\Gamma_{1} f\right], \quad f \in \operatorname{dom}(T),
$$

(i.e., $f=\left(f_{+}, f_{-}\right)^{\top}$ with $f_{ \pm} \in H_{\Delta}^{3 / 2}\left(\Omega_{ \pm}\right)$).

In conclusion, we note that boundary triples for elliptic operators in an unbounded external domain $\Omega_{-} \subset \mathbb{R}^{n}$, used as an illustration in the introduction, were studied, for instance, in [7,28]. The third Green formula in this situation and its analog in connection with noncompact Riemannian manifolds $M$ requires additional techniques to be discussed elsewhere.

Acknowledgements We are indebted to Mark Ashbaugh, Damir Kinzebulatov, and Michael Pang for very helpful discussions. 
Open Access This article is distributed under the terms of the Creative Commons Attribution 4.0 International License (http://creativecommons.org/licenses/by/4.0/), which permits unrestricted use, distribution, and reproduction in any medium, provided you give appropriate credit to the original author(s) and the source, provide a link to the Creative Commons license, and indicate if changes were made.

\section{References}

1. Abramowitz, M., Stegun, I.A.: Handbook of Mathematical Functions. Dover, New York (1972)

2. Aronszajn, N.: A unique continuation theorem for solutions of elliptic partial differential equations or inequalities of second order. J. Math. Pures Appl. 36, 235-249 (1957)

3. Aronszajn, N., Krzywicki, A., Szarski, J.: A unique continuation theorem for exterior differential forms on Riemannian manifolds. Ark. Mat. 4, 417-453 (1962)

4. Behrndt, J., Gesztesy, F., Mitrea, M.: Sharp boundary trace theory and Schrödinger operators on bounded Lipschitz domains (in preparation)

5. Behrndt, J., Langer, M.: Boundary value problems for elliptic partial differential operators on bounded domains. J. Funct. Anal. 243, 536-565 (2007)

6. Behrndt, J., Langer, M.: Elliptic operators, Dirichlet-to-Neumann maps and quasi boundary triples. In: Hassi, S., de Snoo, H.S.V., Szafraniec, F.H. (eds.) Operator Methods for Boundary Value Problems. London Mathematical Society Lecture Note Series, vol. 404, pp. 121-160. Cambridge University Press, Cambridge (2012)

7. Behrndt, J., Langer, M., Lotoreichik, V.: Spectral estimates for resolvent differences of self-adjoint elliptic operators. Integral Equ. Oper. Theory 77, 1-37 (2013)

8. Behrndt, J., Micheler, T.: Elliptic differential operators on Lipschitz domains and abstract boundary value problems. J. Funct. Anal. 267, 3657-3709 (2014)

9. Behrndt, J., Rohleder, J.: An inverse problem of Calderón type with partial data. Commun. Part. Diff. Equ. 37, 1141-1159 (2012)

10. Berezanskiŭ, YuM: Selfadjoint Operators in Spaces of Functions of Infinitely Many Variables, Translations of Mathematical Monographs, vol. 63. American Mathematical Society, Providence, RI (1986)

11. Bruk, V.M.: On a class of problems with the spectral parameter in the boundary conditions. Mat. Sbornik 100, 210-216 (1976)

12. Costabel, M.: Boundary integral operators on Lipschitz domains: elementary results. SIAM J. Math. Anal. 19, 613-626 (1988)

13. Calkin, J.W.: Abstract symmetric boundary conditions. Trans. Am. Math. Soc. 45, 369-442 (1939)

14. Derkach, V.A., Hassi, S., Malamud, M.M., de Snoo, H.S.V.: Generalized resolvents of symmetric operators and admissibility. Methods Funct. Anal. Topol. 6, 24-53 (2000)

15. Derkach, V.A., Hassi, S., Malamud, M.M., de Snoo, H.S.V.: Boundary relations and their Weyl families. Trans. Am. Math. Soc. 358, 5351-5400 (2006)

16. Derkach, V.A., Hassi, S., Malamud, M.M., de Snoo, H.S.V.: Boundary relations and generalized resolvents of symmetric operators. Russ. J. Math. Phys. 16, 17-60 (2009)

17. Derkach, V.A., Hassi, S., Malamud, M.M., de Snoo, H.S.V.: Boundary triplets and Weyl functions. Recent developments. In: Hassi, S., de Snoo, H.S.V., Szafraniec, F.H. (eds.) Operator Methods for Boundary Value Problems. London Mathematical Society Lecture Note Series, vol. 404, pp. 161-220. Cambridge University Press, Cambridge (2012)

18. Derkach, V.A., Malamud, M.M.: Generalized resolvents and the boundary value problems for Hermitian operators with gaps. J. Funct. Anal. 95, 1-95 (1991)

19. Derkach, V.A., Malamud, M.M.: The extension theory of Hermitian operators and the moment problem. J. Math. Sci. 73, 141-242 (1995)

20. Filonov, N.: Second-order elliptic equation of divergence form having a compactly supported solution. J. Math. Sci. (N Y) 106, 3078-3086 (2001)

21. Gorbachuk, V.I., Gorbachuk, M.L.: Boundary Value Problems for Operator Differential Equations. Kluwer, Dordrecht (1991)

22. Hofmann, S., Mitrea, M., Taylor, M.: Geometric and transformational properties of Lipschitz domains, Semmes-Kenig-Toro domains, and other classes of finite perimeter domains. J. Geom. Anal. 17, 593647 (2007)

23. Jerison, D., Kenig, C.E.: Unique continuation and absence of positive eigenvalues for Schrödinger operators. Ann. Math. (2) 121, 463-494 (1985) 
24. John, F.: Partial Differential Equations Applied Mathematical Sciences, vol. 1, 4th edn. Springer, New York (1982)

25. Koch, H., Tataru, D.: Carleman estimates and unique continuation for second-order elliptic equations with nonsmooth coefficients. Commun. Pure Appl. Math. 54, 339-360 (2001)

26. Koch, H., Tataru, D.: Sharp counterexamples in unique continuation for second order elliptic equations. J. Reine Angew. Math. 542, 133-146 (2002)

27. Kochubei, A.N.: Extensions of symmetric operators and symmetric binary relations. Math. Notes 17, 25-28 (1975)

28. Malamud, M.M.: Spectral theory of elliptic operators in exterior domains. Russ. J. Math. Phys. 17, 96-125 (2010)

29. McLean, W.: Strongly Elliptic Systems and Boundary Integral Equations. Cambridge University Press, Cambridge (2000)

30. Mitrea, D., Mitrea, I., Mitrea, M., Taylor, M.: The Hodge-Laplacian: Boundary Value Problems on Riemannian Manifolds, Studies in Mathematics, vol. 64. De Gruyter, Boston (2016)

31. Mitrea, D., Mitrea, M., Taylor, M.: Layer Potentials, the Hodge Laplacian and Global Boundary Problems in Non-Smooth Riemannian Manifolds, vol. 150, No. 713. Memoirs of the American Mathematical Society, Providence, RI (2001)

32. Mitrea, I., Mitrea, M.: Multi-Layer Potentials and Boundary Problems for Higher-Order Elliptic Systems in Lipschitz Domains. Lecture Notes in Mathematics, vol. 2063. Springer, Berlin (2013)

33. Mitrea, M., Taylor, M.: Boundary layer methods for Lipschitz domains in Riemannian manifolds. J. Funct. Anal. 163, 181-251 (1999)

34. Mitrea, M., Taylor, M.: Potential theory on Lipschitz domains in Riemannian manifolds: Hölder continuous metric tensors. Comm. Partial Diff. Equ. 25, 1487-1536 (2000)

35. Mitrea, M., Taylor, M.: Potential theory on Lipschitz domains in Riemannian manifolds: SobolevBesov space results and the Poisson problem. J. Funct. Anal. 176, 1-79 (2000)

36. Rellich, F.: Darstellung der Eigenwerte von $\Delta u+\lambda u=0$ durch ein Randintegral. Math. Z. 46, 635-636 (1940)

37. Taylor, M.: Partial Differential Equations, 2nd ed., vol. I-III. Springer, Berlin 1996 (2011)

38. Wolff, T.H.: Recent work on sharp estimates in second-order elliptic unique continuation problems. J. Geom. Anal. 3, 621-650 (1993) 